\title{
Scheme-independent calculations of properties at a conformal infrared fixed point in gauge theories with multiple fermion representations
}

\author{
Thomas A. Ryttov ${ }^{1}$ and Robert Shrock ${ }^{2}$ \\ ${ }^{1} \mathrm{CP} \mathrm{P}^{3}$-Origins, University of Southern Denmark, Campusvej 55, Odense, Denmark \\ ${ }^{2}$ C. N. Yang Institute for Theoretical Physics and Department of Physics and Astronomy, \\ Stony Brook University, Stony Brook, New York 11794, USA
}

(Received 6 September 2018; published 8 November 2018)

\begin{abstract}
In previous work we have presented scheme-independent calculations of physical properties of operators at a conformally invariant infrared fixed point in an asymptotically free gauge theory with gauge group $G$ and $N_{f}$ fermions in a representation $R$ of $G$. Here we generalize this analysis to the case of fermions in multiple representations, focusing on the case of two different representations. Our results include the calculation of the anomalous dimensions of gauge-invariant fermion bilinear operators, and the derivative of the beta function, evaluated at the infrared fixed point. We illustrate our results in an $\mathrm{SU}\left(N_{c}\right)$ gauge theory with $N_{F}$ fermions in the fundamental representation and $N_{A d j}$ fermions in the adjoint representation.
\end{abstract}

DOI: 10.1103/PhysRevD.98.096003

\section{INTRODUCTION}

In this paper we shall consider a vectorial, asymptotically free gauge theory (in four spacetime dimensions, at zero temperature) with gauge group $G$ with massless fermions transforming according to multiple different representations of $G$, which has an exact infrared (IR) fixed point (IRFP) of the renormalization group [1]. For technical simplicity, we will restrict ourselves to two different representations. We thus take the theory to contain $N_{f}$ copies (flavors) of Dirac fermions, denoted $f$, in the representation $R$ of $G$, and $N_{f^{\prime}}$ copies of fermions, denoted $f^{\prime}$, in a different representation $R^{\prime}$ of $G$. In the case in which $f^{\prime}$ transforms according to a self-conjugate representation, the number $N_{f^{\prime}}$ refers equivalently to a theory with $N_{f^{\prime}}$ Dirac fermions or $2 N_{f^{\prime}}$ Majorana fermions and hence in this case $N_{f^{\prime}}$ may take on half-integral as well as integral values. One motivation for such theories is a possible direction for ultraviolet completions of the Standard Model that might help to explain the origin of the generational hierarchy of quark and lepton masses (e.g., [2,3] and references therein), since this hierarchy could be associated with the different Euclidean energy/momentum scales at which fermion condensates form for fermions in different representations of a strongly coupled gauge interaction involving beyond-Standard-Model (BSM) physics. In [3]

Published by the American Physical Society under the terms of the Creative Commons Attribution 4.0 International license. Further distribution of this work must maintain attribution to the author(s) and the published article's title, journal citation, and DOI. Funded by SCOAP ${ }^{3}$. we studied the infrared evolution and phase structure of this type of theory. Here we go beyond Refs. [2,3] in presenting (scheme-independent) calculations of anomalous dimensions of gauge-invariant operators.

We denote the running gauge coupling as $g=g(\mu)$, where $\mu$ is the Euclidean energy/momentum scale at which this coupling is measured. We define $\alpha(\mu)=g(\mu)^{2} /(4 \pi)$. Since the theory is asymptotically free, its properties can be computed reliably in the deep ultraviolet (UV) region at large $\mu$, where the coupling approaches zero. The dependence of $\alpha(\mu)$ on $\mu$ is described by the renormalization-group (RG) beta function, $\beta=d \alpha(\mu) / d t$, where $d t=d \ln \mu$ (the argument $\mu$ will often be suppressed in the notation). We will consider a theory in which the fermion content is such that the RG flow from the UV to the IR ends in an exact IR fixed point, as determined by the zero in the beta function nearest to the origin for physical coupling, denoted $\alpha_{\mathrm{IR}}$. Since $\beta=0$ at $\alpha=\alpha_{\mathrm{IR}}$, the resultant theory in this IR limit is scale-invariant, and is deduced also to be conformally invariant [4].

The properties of the resultant conformal field theory at this IRFP are of considerable importance. Physical quantities defined at the IRFP obviously cannot depend on the scheme used for the regularization and renormalization of the theory. In conventional computations of these quantities, one first writes them as series expansions in powers of the coupling, and then evaluates these series expansions with $\alpha$ set equal to $\alpha_{\mathrm{IR}}$, calculated to a given loop order. These calculations have been performed for anomalous dimensions of gauge-invariant fermion bilinears in a theory with a single fermion representation up to four-loop level [5-7] and to five-loop level [8]. However, as is well known, these conventional (finite-order) series expansions are 
scheme-dependent beyond the leading terms. Indeed, this is a generic property of higher-order calculations in quantum field theory, such as computations in quantum chromodynamics (QCD) used to compare with data from the Fermilab Tevatron and CERN Large Hadron Collider (LHC).

There is thus strong motivation to calculate and analyze series expansions for physical properties at the IRFP which are scheme-independent at each finite order. The fact that makes this possible is simple but powerful. To review this, we first specialize to a theory with $N_{f}$ fermions in a single representation, $R$, of the gauge group $G$. The constraint of asymptotic freedom means that $N_{f}$ must be less than a certain upper $(u)$ bound, denoted $N_{f, u}$. Here and below, we will often formally generalize the number(s) of fermions in one or multiple representations from non-negative integers to non-negative real numbers, with the understanding that for a physical quantity one restricts to integral values. Furthermore, as noted above, if an $f^{\prime}$ fermion transforms according to a self-conjugate representation, then the number $N_{f^{\prime}}$ refers equivalently to a theory with $N_{f^{\prime}}$ Dirac fermions or $2 N_{f^{\prime}}$ Majorana fermions, so that in this case, $N_{f^{\prime}}$ may take on half-integral physical values. As $N_{f}$ approaches $N_{f, u}$ from below, the value of the IRFP, $\alpha_{\mathrm{IR}}$, approaches zero. This means that one can reexpress series expansions for physical quantities at this IRFP in powers of the manifestly scheme-independent variable $[9,10]$

$$
\Delta_{f}=N_{f, u}-N_{f}
$$

In recent work, for theories with $N_{f}$ fermions in a single representation of the gauge group $G$, we have calculated scheme-independent series expansions for the anomalous dimensions of gauge-invariant fermion bilinears and the derivative $d \beta / d \alpha$, both evaluated at the IRFP, to the respective orders $O\left(\Delta_{f}^{4}\right)$ and $O\left(\Delta_{f}^{5}\right)$ [11-19]. These are the highest orders to which these quantities have been calculated. We gave explicit expressions for the case $G=\mathrm{SU}\left(N_{c}\right)$ and $R$ equal to the fundamental, adjoint, and rank-2 symmetric and antisymmetric tensor representations, and for other Lie groups, including orthogonal, symplectic, and exceptional groups.

In this paper we shall generalize our previous schemeindependent series calculations of physical quantities at an IRFP from the case of an asymptotically free gauge theory with $N_{f}$ fermions in a single representation of the gauge group $G$ to the case of fermions in multiple different representations. Specifically, we consider a theory with $N_{f}$ fermions in a representation $R$ of $G$ and $N_{f^{\prime}}$ fermions in a different representation, $R^{\prime}$, of $G$. We present schemeindependent calculations of the anomalous dimensions of gauge-invariant fermion bilinear operators to cubic order in the respective expansion variable $\left[\Delta_{f}\right.$ in Eq. (1.1) for $\bar{f} f$ and $\Delta_{f^{\prime}}$ in Eq. (1.2) for $\left.\bar{f}^{\prime} f^{\prime}\right]$ and to quartic order in
$\Delta_{f}$ and $\Delta_{f^{\prime}}$ for the derivative of the beta function, evaluated at the infrared fixed point.

The condition of asymptotic freedom requires that the value of a certain linear combination of $N_{f^{\prime}}$ and $N_{f}$ must be less than an upper bound given below by Eq. (2.3). For a fixed $N_{f^{\prime}}$, this implies an upper bound denoted as $N_{f}<N_{f, u}$, and for a fixed $N_{f}$, this implies the upper bound $N_{f^{\prime}}<N_{f^{\prime}, u}$ given respectively in Eqs. (2.4) and (2.5) below. For fixed $N_{f^{\prime}}$, as $N_{f}$ approaches $N_{f, u}$ from below, $\alpha_{\mathrm{IR}}$ approaches zero. Therefore, one can rewrite the series expansions for physical quantities as power series in the variable $\Delta_{f}$. The coefficients in these series expansions depend on $N_{f^{\prime}}$. If $\Delta_{f}$ is small, the value of $\alpha_{\mathrm{IR}}$ is also small, so that the resultant IR theory may be inferred to be in a (deconfined) non-Abelian Coulomb phase (NACP), often called the conformal window. Strong evidence for this in the single-representation case comes from fully nonperturbative lattice simulations [20-22]. In the same way, for fixed $N_{f}$, one can rewrite the series expansions for physical quantities as power series in the variable

$$
\Delta_{f^{\prime}}=N_{f^{\prime}, u}-N_{f^{\prime}} .
$$

For a general operator $\mathcal{O}$, we denote the full scaling dimension as $D_{\mathcal{O}}$ and its free-field value as $D_{\mathcal{O} \text {,free. The }}$ anomalous dimension of this operator, denoted $\gamma_{\mathcal{O}}$, is defined via the relation [23]

$$
D_{\mathcal{O}}=D_{\mathcal{O}, \text { free }}-\gamma_{\mathcal{O}}
$$

Let us denote the fermions of type $f$ as $\psi_{i}, i=1, \ldots, N_{f}$ and the fermions of type $f^{\prime}$ as $\chi_{j}, j=1, \ldots, N_{f^{\prime}}$. We shall calculate scheme-independent series expansions for the anomalous dimensions, denoted $\gamma_{\bar{\psi} \mu \mu, \mathrm{IR}}$ and $\gamma_{\bar{\chi} \chi, \mathrm{IR}}$ of the respective (gauge-invariant) fermion bilinears

$$
\bar{\psi} \psi=\sum_{j=1}^{N_{f}} \bar{\psi}_{j} \psi_{j}
$$

and

$$
\bar{\chi} \chi=\sum_{j=1}^{N_{f^{\prime}}} \bar{\chi}_{j} \chi_{j} .
$$

The anomalous dimension of $\bar{\psi} \psi$ is the same as that of the (gauge-invariant) bilinear $\sum_{j, k=1}^{N_{f}} \bar{\psi}_{j} \mathcal{T}_{a} \psi_{k}$, where $\mathcal{T}_{a}$ is a generator of the Lie algebra of $\mathrm{SU}\left(N_{f}\right)$ [24], and we shall use the symbol $\gamma_{\bar{\psi} \psi \text {,IR }}$ to refer to both. An analogous comment applies to $\gamma_{\bar{\chi} \chi}$,IR . We write the scheme-independent series expansions of $\gamma_{\bar{f} f, \text { IR }}$ as

$$
\gamma_{\bar{f} f, \mathrm{IR}}=\sum_{j=1}^{\infty} \kappa_{j}^{(f)} \Delta_{f}^{j}
$$


and

$$
\gamma_{\bar{f}^{\prime} f^{\prime}, \mathrm{IR}}=\sum_{j=1}^{\infty} \kappa_{j}^{\left(f^{\prime}\right)} \Delta_{f^{\prime}}^{j} .
$$

We shall illustrate our general results in an $\mathrm{SU}\left(N_{c}\right)$ gauge theory with $N_{F}$ fermions of type $f$ in the fundamental $(F)$ representation and $N_{A d j}$ fermions of type $f^{\prime}$ in the adjoint $(A d j)$ representation. For this theory we will also use an explicit notation with coefficients $\kappa^{(f)}=\kappa^{(F)}$ and $\kappa^{\left(f^{\prime}\right)}=\kappa^{(A d j)}$.

We shall calculate two equivalent scheme-independent series expansions of the derivative $\beta_{\mathrm{IR}}^{\prime}$. With $N_{f^{\prime}}$ fixed, and $N_{f}$ variable, one may write the series as an expansion in powers of $\Delta_{f}$ :

$$
\beta_{\mathrm{IR}}^{\prime}=\sum_{j=2}^{\infty} d_{j} \Delta_{f}^{j} .
$$

Alternately, one may take $N_{f}$ to be fixed and write $\beta_{\mathrm{IR}}^{\prime}$ as a series expansion in powers of $\Delta_{f^{\prime}}$, as

$$
\beta_{\mathrm{IR}}^{\prime}=\sum_{j=2}^{\infty} \tilde{d}_{j} \Delta_{f^{\prime}}^{j} .
$$

Note that $d_{1}=\tilde{d}_{1}=0$ for all $G$ and fermion representations.

This paper is organized as follows. In Sec. II we discuss the methodology for our calculations. In Secs. III and V we present our new results for scheme-independent expansions of the anomalous dimensions of fermion bilinears and $d \beta / d \alpha$, both evaluated at the infrared fixed point. We discuss the special cases of the anomalous dimension and $\beta_{\mathrm{IR}}^{\prime}$ results for an illustrative theory with gauge group $\mathrm{SU}\left(N_{c}\right)$ containing fermions in the fundamental and adjoint representations in Secs. IV and VI, respectively. Our conclusions are given in Sec. VII, and some relevant group-theoretic results are reviewed in Appendix.

\section{CALCULATIONAL METHODS}

\section{A. Beta function and series expansions for physical quantities}

In this section we discuss some background and the calculational methods that are relevant for our present work. The series expansion of $\beta$ in powers of the squared gauge coupling is

$$
\beta=-2 \alpha \sum_{\ell=1}^{\infty} b_{\ell} a^{\ell}
$$

where $a=g^{2} /\left(16 \pi^{2}\right)=\alpha /(4 \pi)$ and $b_{\ell}$ is the $\ell$-loop coefficient. With an overall minus sign extracted, as in Eq. (2.1), the condition of asymptotic freedom is that $b_{1}>0$. The one-loop coefficient, $b_{1}$, is independent of the scheme used for regularization and renormalization. Massindependent schemes include minimal subtraction [25] and modified minimal subtraction, denoted $\overline{\mathrm{MS}}$ [26]. For massindependent schemes, the two-loop coefficient, $b_{2}$, is also independent of the specific scheme used [27]. For a theory with a general gauge group $G$ and $N_{f}$ fermions in a single representation, $R$, the coefficients $b_{1}$ and $b_{2}$ were calculated in [28] and [29], while $b_{3}, b_{4}$, and $b_{5}$ were calculated in the commonly used $\overline{\mathrm{MS}}$ scheme in [30], [31], and [32], respectively (see also [33]). For the analysis of a theory with fermions in multiple different representations, one needs generalizations of these results. These are straightforward to derive in the case of $b_{1}$ and $b_{2}$, but new calculations are required for higher-loop coefficients. These have recently been performed in [34] (again in the $\overline{\mathrm{MS}}$ scheme) up to four-loop order, and we use the results of Ref. [34] here.

The expansion of the anomalous dimension of the fermion bilinear $\gamma_{\bar{\psi} \psi}$ in powers of the squared gauge coupling is

$$
\gamma_{\bar{\psi} \psi \psi}=\sum_{\ell=1}^{\infty} c_{\ell}^{(f)} a^{\ell},
$$

where $c_{\ell}^{(f)}$ is the $\ell$-loop coefficient. The analogous expansion applies for $\gamma_{\bar{\chi} \chi}$ with the replacement $c_{\ell}^{(f)} \rightarrow c_{\ell}^{\left(f^{\prime}\right)}$. The one-loop coefficient $c_{1}^{(f)}$ is scheme-independent, while the $c_{\ell}^{(f)}$ with $\ell \geq 2$ are scheme-dependent, and similarly with the $c_{\ell}^{\left(f^{\prime}\right)}$. For a general gauge group $G$ and $N_{f}$ fermions in a single representation $R$ of $G$, the $c_{\ell}^{(f)}$ have been calculated up to loop order $\ell=4$ in [35] and $\ell=5$ in [36]. For the case of multiple fermion representations, the anomalous dimension coefficients for the fermion bilinears have been calculated up to four-loop order in [37]. We use the results of [37] up to three-loop order here.

Concerning scheme-independent series expansions, the calculation of the coefficient $\kappa_{j}^{(f)}$ in Eq. (1.6) requires, as inputs, the values of the $b_{\ell}$ for $1 \leq \ell \leq j+1$ and the $c_{\ell}^{(f)}$ for $1 \leq \ell \leq j$, and similarly for $\kappa_{j}^{\left(f^{\prime}\right)}$, with the replacement $c_{\ell}^{(f)} \rightarrow c_{\ell}^{\left(f^{\prime}\right)}$. The calculation of the coefficients $d_{j}$ and $\tilde{d}_{j}$ in Eqs. (1.8) and (1.9) requires, as inputs, the values of the $b_{\ell}$ for $1 \leq \ell \leq j$.

Thus, using the calculation of the beta function for multiple fermion representation to four-loop order in [34], together with the calculation of the anomalous dimensions of the fermion bilinears in [37] up to three-loop order, we can calculate $\gamma_{\bar{\psi} \psi, \text { IR }}$ to order $O\left(\Delta_{f}^{3}\right)$ and $\gamma_{\bar{\chi} \chi, \text { IR }}$ to $O\left(\Delta_{f^{\prime}}^{3}\right)$ for the case of multiple fermion representations. [Note that we cannot make use of the four-loop calculation of the anomalous dimensions of fermion bilinears in [37] to 
compute $\gamma_{\bar{\psi} \psi, \mathrm{IR}}$ to order $O\left(\Delta_{f}^{4}\right)$ and $\gamma_{\bar{\chi} \chi, \mathrm{IR}}$ to $O\left(\Delta_{f^{\prime}}^{4}\right)$, because this would require, as an input, the five-loop coefficient $b_{5}$ in the beta function for this case of multiple fermion representations, and, to our knowledge, this has not been calculated.]

Similarly, using the four-loop beta function from [34], we can calculate the $d_{j}$ and $\tilde{d}_{j}$ for $\beta_{\mathrm{IR}}^{\prime}$ to order $j=4$. We denote the truncation of these series to maximal power $j=p$ as $\gamma_{\bar{\psi} \mu, \mathrm{IR}, \Delta_{f}^{p}}, \gamma_{\bar{\chi} \chi, \mathrm{IR}, \Delta_{f \prime}^{p}}, \beta_{\mathrm{IR}, \Delta_{f}^{p}}^{\prime}$, and $\beta_{\mathrm{IR}, \Delta_{f^{\prime}}^{p}}^{\prime}$, respectively. Although we use these coefficients as calculated in the $\overline{\mathrm{MS}}$ scheme below, we emphasize that our results are schemeindependent, so the specific scheme used for their calculation does not matter. An explicit illustration of this using several schemes is given in [38]. We refer the reader to our previous work for detailed discussions of the procedure for calculating the coefficients $\kappa_{j}$ and $d_{j}$ in the case of a theory with $N_{f}$ fermions in a single representation of $G$.

Our procedure for calculating scheme-independent series expansions requires that the IRFP be exact, and hence we restrict our consideration to the non-Abelian Coulomb phase, where this condition is satisfied. For sufficiently smaller values of $N_{f}$ and/or $N_{f^{\prime}}$, there is spontaneous chiral symmetry breaking $(\mathrm{S} \chi \mathrm{SB})$, giving rise to dynamical masses for the $f$ and/or $f^{\prime}$ fermions [39]. Most-attractive channel arguments suggest that as $N_{f}$ and/ or $N_{f^{\prime}}$ decrease(s) and $\alpha_{\mathrm{IR}}$ increases, the fermion with the largest value of $C_{f}$ would be the first to form bilinear fermion condensates and hence obtain dynamical masses and be integrated out of the low-energy effective field theory (EFT). Assuming that this happens and, say, the $f^{\prime}$ fermions condense out, then one would proceed to examine the resultant EFT with the remaining massless $f$ fermions to determine the further evolution of this theory into the infrared. The details of the construction of the EFT will not be relevant here, since we restrict our analysis to the (chirally symmetric) non-Abelian Coulomb phase.

\section{B. Relevant range of $\left(N_{f}, N_{f^{\prime}}\right)$}

Since we require that the theory should be asymptotically free and since our scheme-independent calculational method requires an exact IR fixed point, which is satisfied in the non-Abelian Coulomb phase, a first step is to discuss the corresponding values of the pair $\left(N_{f}, N_{f^{\prime}}\right)$ that satisfy these conditions. We denote this set of values, or more generally, the region in the first quadrant of the $\mathbb{R}^{2}$ plane defined by the generalization of $\left(N_{f}, N_{f^{\prime}}\right)$ from nonnegative integers (or half-integers in the case of a Majorana fermion in a self-conjugate representation) to non-negative real numbers, where the theory has an IRFP in the non-Abelian Coulomb phase as the region $\mathcal{R}_{\mathrm{NACP}}$. We next discuss the boundaries of this region.

For a specified gauge group $G$ and fermion representations $R$ and $R^{\prime}$, the numbers $N_{f}$ and $N_{f^{\prime}}$ are bounded above by the asymptotic freedom (AF) condition that $b_{1}>0$. This condition is expressed as the inequality on the linear combination

$$
N_{f} T_{f}+N_{f^{\prime}} T_{f^{\prime}}<\frac{11 C_{A}}{4},
$$

where $C_{A}$ and $T_{f}$ are group invariants defined in Appendix. Thus, for fixed $N_{f^{\prime}}$, the AF property implies that $N_{f}$ is bounded above as $N_{f}<N_{f, u}$, where

$$
N_{f, u}=\frac{11 C_{A}-4 N_{f^{\prime}} T_{f^{\prime}}}{4 T_{f}}
$$

and similarly, for fixed $N_{f}$, the AF condition implies that $N_{f^{\prime}}$ is bounded above as $N_{f^{\prime}}<N_{f^{\prime}, u}$, where

$$
N_{f^{\prime}, u}=\frac{11 C_{A}-4 N_{f} T_{f}}{4 T_{f^{\prime}}} .
$$

The upper boundary of this asymptotically free region, which is also the upper boundary of the region $\mathcal{R}_{\mathrm{NACP}}$, in $N_{f}$ and $N_{f^{\prime}}$ is the locus of solutions to the condition $b_{1}=0$. This is a finite segment of the line $N_{f} T_{f}+N_{f^{\prime}} T_{f^{\prime}}=11 C_{A} / 4$. We may picture the first quadrant in the $\mathbb{R}^{2}$ space defined by non-negative $\left(N_{f}, N_{f^{\prime}}\right)$ to be such that $N_{f}$ is the horizontal axis and $N_{f^{\prime}}$ is the vertical axis. Then the line segment bounding the asymptotically free region is an oblique line segment running from the upper left to the lower right, with slope

$$
\left.\frac{\partial N_{f^{\prime}}}{\partial N_{f}}\right|_{b_{1}=0}=-\frac{T_{f}}{T_{f^{\prime}}}
$$

This line segment intersects the horizontal axis at the point $\left(N_{f}, N_{f^{\prime}}\right)=\left(11 C_{A} /\left(4 T_{f}\right), 0\right)$ and the vertical axis at the point $\left(N_{f}, N_{f^{\prime}}\right)=\left(0,11 C_{A} /\left(4 T_{f^{\prime}}\right)\right)$. Without loss of generality, we take $f$ to be the (nonsinglet) fermion representation of smaller dimension. The respective schemeindependent expansions in powers of $\Delta_{f}$ and $\Delta_{f^{\prime}}$ amount to moving into the interior of the non-Abelian Coulomb phase from the upper boundary line horizontally (moving leftward) and vertically (moving downward).

In our earlier work on theories with $N_{f}$ fermions in a single fermion representation of the gauge group, we denoted the lower boundary of the NACP as $N_{f, c r}$. In that case, we assumed that $N_{f}$ was in the NACP interval $I_{\mathrm{NACP}}: N_{f, c r}<N_{f}<N_{f, u}$. Here the generalization of this is the set of physical values of $N_{f}$ and $N_{f^{\prime}}$ in the region $\mathcal{R}_{\mathrm{NACP}}$. Even in the case of a single fermion representation, the value of $N_{f, c r}$ is not known precisely. This question of the value of $N_{f, c r}$ for various specific theories has been investigated in a number of lattice studies [20,21], which continue at present. As noted above, we have previously 
presented approximate analytic results relevant for this study in [2,3]. Corresponding lattice studies could be carried out for theories with multiple different fermion representations to study properties of the respective theories. An example is a recent lattice study of an SU(4) gauge theory with $N_{f}=2$ Dirac fermions in the fundamental representation and $N_{f^{\prime}}=2$ Dirac fermions in the (selfconjugate) antisymmetric rank-2 tensor representation $[40,41]$, which finds that the (zero-temperature) theory is in the phase with chiral symmetry breaking for both types of fermions. Since our results are restricted to an exact infrared fixed point in the (conformally invariant) nonAbelian Coulomb phase, they are not directly applicable to this theory.

For the present study, with the axes of the first-quadrant quarter plane in $\left(N_{f}, N_{f^{\prime}}\right) \in \mathbb{R}^{2}$ as defined above, the upper boundary of the NACP is the line segment resulting from the $b_{1}=0$ condition. The analogue of the lower boundary of the NACP at $N_{f, c r}$ for the present study with two fermion representations is a line segment or nonlinear curve displaced in the direction to the lower left relative to the oblique $b_{1}=0$ line, so that the resultant NACP forms a region in which physical values of $N_{f}$ and $N_{f^{\prime}}$ define possible IR theories. This lower boundary of the NACP intersects the horizontal axis at the point $\left(N_{f}, N_{f^{\prime}}\right)=$ $\left(N_{f, c r}, 0\right)$ and intersects the vertical axis at the point $\left(N_{f}, N_{f^{\prime}}\right)=\left(0, N_{f^{\prime}, c r}\right)$. Although this lower boundary of the NACP is not known, one can get a rough idea of where it lies by generalizing the analysis that we gave in our previous work for theories with a single fermion representation $[12,13,15]$. This analysis was based on the observation that the two-loop beta function has an IR zero if $N_{f}$ is sufficiently large that $b_{2}$ is negative (with $b_{1}>0$ ). In this case of a single fermion representation, for small $N_{f}$, $b_{2}$ is positive, and turns negative when $N_{f}$ exceeds a certain lower $(\ell)$ value $N_{f, \ell}<N_{f, u}$ where $b_{2}=0$, namely

$$
N_{f, \ell}=\frac{17 C_{A}^{2}}{2 T_{f}\left(5 C_{A}+3 C_{f}\right)} \quad\left(\text { for } N_{f^{\prime}}=0\right) .
$$

Thus, in this single-representation case, if and only if $N_{f}$ lies in an interval that we have denoted previously as $I_{\text {IRZ }}$, the two-loop beta function has an IR zero (IRZ). This interval $I_{\mathrm{IRZ}}$ is

$$
I_{\text {IRZ }}: N_{f, \ell}<N_{f}<N_{f, u} \quad\left(\text { for } N_{f^{\prime}}=0\right) .
$$

Although $N_{f, \ell}$ is not, in general, equal to $N_{f, c r}$, it is moderately close to the latter in theories that have been studied. As an example, in the case of an $\mathrm{SU}\left(N_{c}\right)$ gauge theory with $N_{f}$ fermions in the fundamental $(F)$ representation,

$$
\mathrm{SU}\left(N_{c}\right), R=F: N_{f, \ell}=\frac{34 N_{c}^{3}}{13 N_{c}^{2}-3} .
$$

In the intensively studied case $N_{c}=3$ theory, $N_{\ell}=$ $153 / 19 \simeq 8.05$. This is close to the estimates of $N_{f, c r}$ for this theory from our previous studies and from a number of lattice simulations [12,15,20,21].

In our present asymptotically free theory with two fermion representations, the two-loop beta function has an IR zero if and only if $b_{2}<0$, which is the inequality

$N_{f} T_{f}\left(5 C_{A}+3 C_{f}\right)+N_{f^{\prime}} T_{f^{\prime}}\left(5 C_{A}+3 C_{f^{\prime}}\right)>\frac{17 C_{A}^{2}}{2}$.

This IR zero of the two-loop $(2 \ell)$ beta function occurs at $\alpha=\alpha_{\mathrm{IR}, 2 \ell}$, where

$$
\alpha_{\mathrm{IR}, 2 \ell}=-\frac{4 \pi b_{1}}{b_{2}}=\frac{2 \pi\left[11 C_{A}-4\left(N_{f} T_{f}+N_{f^{\prime}} T_{f^{\prime}}\right)\right]}{\left[2 N_{f} T_{f}\left(5 C_{A}+3 C_{f}\right)+2 N_{f^{\prime}} T_{f^{\prime}}\left(5 C_{A}+3 C_{f^{\prime}}\right)-17 C_{A}^{2}\right]}
$$

We thus define the two-dimensional region in the first quadrant of the $\mathbb{R}^{2}$ plane defined by non-negative real values of $\left(N_{f}, N_{f^{\prime}}\right)$ where the theory is asymptotically free and the two-loop beta function has an IR zero as the

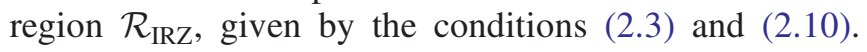
The upper boundary of $\mathcal{R}_{\mathrm{IRZ}}$ is the same as the upper boundary of $\mathcal{R}_{\mathrm{NACP}}$, while the lower boundary of $\mathcal{R}_{\mathrm{IRZ}}$ can provide a rough guide to the lower boundary of $\mathcal{R}_{\text {NACP }}$ and has the advantage that it is exactly calculable. This lower boundary of the region $\mathcal{R}_{\mathrm{IRZ}}$ is given by the solution of the condition that $b_{2}=0$ in the first quadrant of the $\mathbb{R}^{2}$ plane. This condition is obtained from Eq. (2.10) by replacing the inequality by an equality.
The corresponding line defining the lower boundary of $\mathcal{R}_{\text {IRZ }}$ has the slope

$$
\left.\frac{\partial N_{f^{\prime}}}{\partial N_{f}}\right|_{b_{2}=0}=-\frac{T_{f}\left(5 C_{A}+3 C_{f}\right)}{T_{f^{\prime}}\left(5 C_{A}+3 C_{f^{\prime}}\right)}
$$

This lower boundary of the region $\mathcal{R}_{\mathrm{IRZ}}$ crosses the horizontal axis in the $\left(N_{f}, N_{f^{\prime}}\right)$ space at the point $\left(N_{f, \ell}, 0\right)$, where $N_{f, \ell}$ was given above in Eq. (2.7), and it crosses the vertical axis at the corresponding value $\left(0, N_{f^{\prime}, \ell}\right)$, where 


$$
N_{f^{\prime}, \ell}=\frac{17 C_{A}^{2}}{2 T_{f^{\prime}}\left(5 C_{A}+3 C_{f^{\prime}}\right)} .
$$

As noted, the lower boundary of this $\mathcal{R}_{\mathrm{IRZ}}$ region provides a rough guide to the actual lower boundary of the NACP region $\mathcal{R}_{\mathrm{NACP}}$. The determination of the true lower boundary of $\mathcal{R}_{\mathrm{NACP}}$ would require a fully nonperturbative analysis, e.g., via lattice simulations.

Although our calculational methods require the IRFP to be exact and hence, strictly speaking, apply only in the nonAbelian Coulomb phase, they could also be useful for the investigation of quasi-conformal gauge theories. In turn, the latter have been of interest as possible ultraviolet completions of the Standard Model. Specifically, (a) if the transition from the lower part of the non-Abelian Coulomb phase to the quasi-conformal regime in the variables $\left(N_{f}, N_{f^{\prime}}\right)$ is continuous, and (b) if our series calculations are sufficiently accurate in this region, our results for $\gamma_{\bar{\psi} \psi \mu, \mathrm{IR}}, \gamma_{\bar{\chi} \chi, \mathrm{IR}}$, and $\beta_{\mathrm{IR}}^{\prime}$ could provide approximate estimates for the values of these quantities in the quasi-conformal regime just below the lower boundary with the NACP. Here we recall that anomalous dimensions of fermion bilinears in strongly coupled BSM theories are relevant in helping to determine resultant SM fermion masses (e.g., [2,3,20,21]). Another example with fermions in multiple representations is supersymmetric gauge theories with both gauginos and matter chiral superfields.

\section{Example with fermions in the fundamental and adjoint representations}

As an illustrative example, we consider a theory with the gauge group $\mathrm{SU}\left(N_{c}\right)$ that contains $N_{f} \equiv N_{F}$ fermions in the fundamental $(F)$ representation and $N_{f^{\prime}} \equiv N_{A d j}$ fermions in the adjoint representation, $A d j$. We denote this as the FA theory. Here the upper boundary of the NACP region $\mathcal{R}_{\mathrm{NACP}}$, which is also the upper boundary of the region $\mathcal{R}_{\mathrm{IRZ}}$, is given by the line

$$
\text { FA theory: } N_{F}+2 N_{c} N_{A d j}=\frac{11 N_{c}}{2} \text {. }
$$

Thus, $N_{F}<(11 / 2) N_{c}$ if $N_{A d j}=0$ and $N_{A d j}<11 / 4=$ 2.75 if $N_{f}=0$. The lower boundary of $\mathcal{R}_{\mathrm{IRZ}}$, which can provide an approximate estimate to the lower boundary of $\mathcal{R}_{\mathrm{NACP}}$, is given by the line $b_{2}=0$, namely

FA theory: $\left(13 N_{c}-3 N_{c}^{-1}\right) N_{F}+32 N_{c}^{2} N_{A d j}=34 N_{c}^{2}$.

Thus, in $\mathcal{R}_{\mathrm{IRZ}}$, it follows that $N_{F}>34 N_{c}^{3} /\left(13 N_{c}^{2}-3\right)$ if $N_{A d j}=0$ and $N_{A d j}>17 / 16=1.0625$ if $N_{F}=0$. In this FA theory, the line $b_{1}=0$ has slope
TABLE I. List of asymptotically free SU(3) gauge theories with $N_{F}$ fermions in the fundamental $(F)$ representation and $N_{A d j}$ fermions in the adjoint $(A d j)$ representation, with the property that the two-loop beta function has an IR zero, at $\alpha=\alpha_{\mathrm{IR}, 2 \ell}$. The four columns list $\left(N_{F}, N_{A d j}\right), d_{u}, d_{\ell}$, and $\alpha_{\mathrm{IR}, 2 \ell}$, where $d_{u}$ and $d_{\ell}$ are the distances of the point $\left(N_{F}, N_{A d j}\right)$ to the line $b_{1}=0$ and to the line $b_{2}=0$, respectively. Half-integral values of $N_{A d j}$ correspond to

\begin{tabular}{|c|c|c|c|}
\hline$\left(N_{F}, N_{A d j}\right)$ & $d_{u}$ & $d_{\ell}$ & $\alpha_{\mathrm{IR}, 2 \ell}$ \\
\hline$(0,3 / 2)$ & 1.233 & 0.434 & 1.496 \\
\hline$(0,2)$ & 0.740 & 0.929 & 0.419 \\
\hline$(0,5 / 2)$ & 0.247 & 1.425 & 0.0911 \\
\hline$(1,1)$ & 1.562 & 0.0688 & 11.938 \\
\hline$(1,3 / 2)$ & 1.069 & 0.565 & 0.996 \\
\hline$(1,2)$ & 0.575 & 1.060 & 0.286 \\
\hline$(1,5 / 2)$ & 0.0822 & 1.556 & 0.0278 \\
\hline$(2,1)$ & 1.397 & 0.200 & 3.683 \\
\hline$(2,3 / 2)$ & 0.904 & 0.695 & 0.684 \\
\hline$(2,2)$ & 0.411 & 1.191 & 0.182 \\
\hline$(3,1)$ & 1.233 & 0.330 & 1.963 \\
\hline$(3,3 / 2)$ & 0.740 & 0.826 & 0.471 \\
\hline$(3,2)$ & 0.247 & 1.322 & 0.0982 \\
\hline$(4,1)$ & 1.069 & 0.461 & 1.219 \\
\hline$(4,3 / 2)$ & 0.575 & 0.957 & 0.316 \\
\hline$(4,2)$ & 0.0822 & 1.453 & 0.0298 \\
\hline$(5,1 / 2)$ & 1.397 & 0.0964 & 7.630 \\
\hline$(5,1)$ & 0.904 & 0.592 & 0.804 \\
\hline$(5,3 / 2)$ & 0.411 & 1.088 & 0.199 \\
\hline$(6,1 / 2)$ & 1.233 & 0.227 & 2.856 \\
\hline$(6,1)$ & 0.740 & 0.723 & 0.539 \\
\hline$(6,3 / 2)$ & 0.247 & 1.219 & 0.106 \\
\hline$(7,1 / 2)$ & 1.069 & 0.358 & 1.571 \\
\hline$(7,1)$ & 0.575 & 0.854 & 0.355 \\
\hline$(7,3 / 2)$ & 0.0822 & 1.349 & 0.0321 \\
\hline$(8,1 / 2)$ & 0.904 & 0.489 & 0.973 \\
\hline$(8,1)$ & 0.411 & 0.985 & 0.220 \\
\hline$(9,0)$ & 1.233 & 0.124 & 5.236 \\
\hline$(9,1 / 2)$ & 0.740 & 0.620 & 0.628 \\
\hline$(9,1)$ & 0.247 & 1.115 & 0.116 \\
\hline$(10,0)$ & 1.069 & 0.255 & 2.208 \\
\hline$(10,1 / 2)$ & 0.575 & 0.750 & 0.4035 \\
\hline$(10,1)$ & 0.0822 & 1.246 & 0.0347 \\
\hline$(11,0)$ & 0.904 & 0.386 & 1.234 \\
\hline$(11,1 / 2)$ & 0.411 & 0.881 & 0.245 \\
\hline$(12,0)$ & 0.740 & 0.516 & 0.754 \\
\hline$(12,1 / 2)$ & 0.247 & 1.012 & 0.128 \\
\hline$(13,0)$ & 0.575 & 0.647 & 0.468 \\
\hline$(13,1 / 2)$ & 0.0822 & 1.143 & 0.03785 \\
\hline$(14,0)$ & 0.411 & 0.778 & 0.278 \\
\hline$(15,0)$ & 0.247 & 0.909 & 0.143 \\
\hline$(16,0)$ & 0.0822 & 1.040 & 0.0416 \\
\hline
\end{tabular}
$2 N_{A d j}$ copies of Majorana fermions in the adjoint representation. 


$$
\text { FA theory: }\left.\frac{\partial N_{A d j}}{\partial N_{F}}\right|_{b_{1}=0}=-\frac{1}{2 N_{c}},
$$

while the line $b_{2}=0$ has slope

$$
\text { FA theory: }\left.\frac{\partial N_{A d j}}{\partial N_{F}}\right|_{b_{2}=0}=-\frac{\left(13 N_{c}^{2}-3\right)}{32 N_{c}^{3}} .
$$

For example, in the FA theory with $N_{c}=3$, so $G=\mathrm{SU}(3)$, these slopes (2.16) and (2.17) are $-1 / 6=$ -0.16667 and $-19 / 144=-0.13194$, respectively, where the floating-point values are given to the indicated accuracy. The $b_{1}=0$ line crosses the horizontal and vertical axes at $\left(N_{f}, N_{f^{\prime}}\right)=(16.5,0)$ and $(0,2.75)$, respectively, while the $b_{2}=0$ line crosses the horizontal and vertical axes at $\left(N_{f}, N_{f^{\prime}}\right)=(8.0526,0)$ and $(0,1.0625)$, respectively.

In Table I we list the physical integral values of $N_{F}$ and integral and half-integral (Majorana) values of $N_{A d j}$ in the region $\mathcal{R}_{\mathrm{IRZ}}$ in this $\mathrm{SU}(3)$ theory. Considering $\left(N_{F}, N_{\text {Adj }}\right)$ as a point in the first quadrant of an $\mathbb{R}^{2}$ space, we list in the second column the distance $d_{u}$ of this point from the line $b_{1}=0$ that forms the upper boundary of the regions $\mathcal{R}_{\mathrm{IRZ}}$ and $\mathcal{R}_{\mathrm{NACP}}$, and in the third column the distance $d_{\ell}$ of this point from the line $b_{2}=0$ that forms the lower boundary of the region $\mathcal{R}_{\mathrm{IRZ}}$. (By distance of a point $P$ from a line $L$, we mean the length of the line segment perpendicular to the line $L$ that passes through the point $P$.) Thus, Table I provides a guide to the position of a theory with a given set of values of $\left(N_{F}, N_{A d j}\right)$ in the region $\mathcal{R}_{\mathrm{IRZ}}$. In general, theories with small values of $d_{u}$ are close to the upper boundary of the region $\mathcal{R}_{\mathrm{NACP}}$ and have correspondingly small values of $\alpha_{\mathrm{IR}}$. In order for our perturbative analysis to be self-consistent, it is necessary that $\alpha_{\mathrm{IR}}$ should not be excessively large, and so one may require, say, that $\alpha_{\mathrm{IR}, 2 \ell}<1$. Our perturbative analysis is expected to be most accurate for the $\left(N_{F}, N_{A d j}\right)$ FA theories with small $d_{u}$ and hence small $\alpha_{\mathrm{IR}, 2 \ell}$ in the upper part of the NACP. We will discuss this illustrative two-representation FA theory further below.

\section{SCHEME-INDEPENDENT CALCULATION OF ANOMALOUS DIMENSIONS OF FERMION BILINEAR OPERATORS}

In this section, for a theory with a general gauge group $G$ containing $N_{f}$ fermions in a representation $R$ and $N_{f^{\prime}}$ fermions in a representation $R^{\prime}$, we present our new calculations of the coefficients $\kappa_{j}^{(f)}$ and $\kappa_{j}^{\left(f^{\prime}\right)}$ in the scheme-independent expansions of the anomalous dimensions $\gamma_{\bar{\psi} \psi, \text { IR }}$ and $\gamma_{\bar{\chi} \chi, \text { IR }}$ in Eqs. (1.6) and the analogue for $\gamma_{\bar{\chi} \chi, I R}$ with $1 \leq j \leq 3$. It will be useful to define a factor that occurs repeatedly in the denominators of various expressions, namely

$$
\mathcal{D}_{f}=C_{A}\left(7 C_{A}+11 C_{f}\right)+4 N_{f^{\prime}} T_{f^{\prime}}\left(C_{f^{\prime}}-C_{f}\right) .
$$

In the previously studied theory with a single fermion representation, i.e., $N_{f^{\prime}}=0$, this factor $\mathcal{D}$ reduces as

$$
\mathcal{D}_{f}=C_{A} D \quad \text { if } N_{f^{\prime}}=0
$$

where

$$
D=7 C_{A}+11 C_{f},
$$

as defined in Eq. (2.13) of our earlier work $[13,15]$.

For the first two coefficients we calculate

$$
\kappa_{1}^{(f)}=\frac{8 C_{f} T_{f}}{\mathcal{D}_{f}}
$$

and

$$
\begin{aligned}
\kappa_{2}^{(f)}= & \frac{4 C_{f} T_{f}^{2}}{3 \mathcal{D}_{f}^{3}}\left[C_{A}\left(7 C_{A}+4 C_{f}\right)\left(5 C_{A}+88 C_{f}\right)\right. \\
& \left.+2^{4} N_{f^{\prime}} T_{f^{\prime}}\left(C_{f^{\prime}}-C_{f}\right)\left(10 C_{A}+8 C_{f}+C_{f^{\prime}}\right)\right] .
\end{aligned}
$$

For the third coefficient, we write

$\kappa_{3}^{(f)}=\frac{4 C_{f} T_{f}}{3^{4} \mathcal{D}_{f}^{5}}\left[A_{0}^{(f)}+A_{1}^{(f)} N_{f^{\prime}}+A_{2}^{(f)} N_{f^{\prime}}^{2}+A_{3}^{(f)} N_{f^{\prime}}^{3}\right]$.

It follows that the $A_{0}^{(f)}$ term is independent of $N_{f^{\prime}}$ and hence, taking into account the difference in the prefactor, it is equal to $C_{A}$ times the terms in the square bracket of Eq. (6.7) in our earlier Ref. [13] or equivalently Eq. (3.4) in our Ref. [15]. We have

$$
\begin{aligned}
A_{0}^{(f)}= & C_{A}\left[3 C_{A} T_{f}^{2}\left(-18473 C_{A}^{4}+144004 C_{A}^{3} C_{f}+650896 C_{A}^{2} C_{f}^{2}+356928 C_{A} C_{f}^{3}+569184 C_{f}^{4}\right)\right. \\
& +2^{7} D\left(-20 T_{f}^{2} \frac{d_{A}^{a b c d} d_{A}^{a b c d}}{d_{A}}+352 C_{A} T_{f} \frac{d_{f}^{a b c d} d_{A}^{a b c d}}{d_{A}}-1331 C_{A}^{2} \frac{d_{f}^{a b c d} d_{f}^{a b c d}}{d_{A}}\right) \\
& \left.+33 \cdot 2^{10} D \zeta_{3}\left(2 T_{f}^{2} \frac{d_{A}^{a b c d} d_{A}^{a b c d}}{d_{A}}-13 C_{A} T_{f} \frac{d_{f}^{a b c d} d_{A}^{a b c d}}{d_{A}}+11 C_{A}^{2} \frac{d_{f}^{a b c d} d_{f}^{a b c d}}{d_{A}}\right)\right]
\end{aligned}
$$


where $\zeta_{s}=\sum_{n=1}^{\infty} n^{-s}$ is the Riemann zeta function. Here, the group invariants $C_{A}, C_{f}, T_{f}, d_{A}^{a b c d} d_{A}^{a b c d}, d_{f}^{a b c d} d_{A}^{a b c d}$, $d_{f}^{a b c d} d_{f}^{a b c d}$, and $d_{f}^{a b c d} d_{f^{\prime}}^{a b c d}$ are defined in the Appendix, and $d_{A}$ is the dimension of the adjoint representation of $G$.

For the other $A_{s}^{(f)}$ with $1 \leq s \leq 3$, we calculate

$$
\begin{aligned}
& A_{1}^{(f)}=C_{A} T_{f}^{2} T_{f^{\prime}}\left[273840 C_{A}^{3}\left(C_{f^{\prime}}-C_{f}\right)+C_{A}^{2}\left(-1511040 C_{f}^{2}+1916256 C_{f} C_{f^{\prime}}-405216 C_{f^{\prime}}^{2}\right)\right. \\
& +C_{A}\left(-129600 C_{f}^{3}+522432 C_{f}^{2} C_{f^{\prime}}-485568 C_{f} C_{f^{\prime}}^{2}+92736 C_{f^{\prime}}^{3}\right) \\
& \left.+C_{f}\left(-1241856 C_{f}^{3}+1020096 C_{f}^{2} C_{f^{\prime}}+76032 C_{f} C_{f^{\prime}}^{2}+145728 C_{f^{\prime}}^{3}\right)\right] \\
& +10240 T_{f}^{2} T_{f^{\prime}}\left(C_{f}-C_{f^{\prime}}\right) \frac{d_{A}^{a b c d} d_{A}^{a b d c}}{d_{A}}+C_{A} T_{f} T_{f^{\prime}}\left(-114688 C_{A}-360448 C_{f}+180224 C_{f^{\prime}}\right) \frac{d_{f}^{a b c d} d_{A}^{a b c d}}{d_{A}} \\
& +C_{A} T_{f}^{2}\left(114688 C_{A}+180224 C_{f}\right) \frac{d_{f^{\prime}}^{a b c d} d_{A}^{a b c d}}{d_{A}}+C_{A}^{2} T_{f^{\prime}}\left(867328 C_{A}+2044416 C_{f}-681472 C_{f^{\prime}}\right) \frac{d_{f}^{a b c d} d_{f}^{a b c d}}{d_{A}} \\
& +C_{A}^{2} T_{f}\left(-867328 C_{A}-1362944 C_{f}\right) \frac{d_{f}^{a b c d} d_{f^{\prime}}^{a b c d}}{d_{A}} \\
& +\zeta_{3}\left[270336 T_{f}^{2} T_{f^{\prime}}\left(C_{f^{\prime}}-C_{f}\right) \frac{d_{A}^{a b c d} d_{A}^{a b c d}}{d_{A}}+C_{A} T_{f} T_{f^{\prime}}\left(1118208 C_{A}+3514368 C_{f}-1757184 C_{f^{\prime}}\right) \frac{d_{f}^{a b c d} d_{A}^{a b c d}}{d_{A}}\right. \\
& +C_{A} T_{f}^{2}\left(-1118208 C_{A}-1757184 C_{f}\right) \frac{d_{f^{\prime}}^{a b c d} d_{A}^{a b c d}}{d_{A}}+C_{A}^{2} T_{f^{\prime}}\left(-1892352 C_{A}-4460544 C_{f}+1486848 C_{f^{\prime}}\right) \frac{d_{f}^{a b c d} d_{f}^{a b c d}}{d_{A}} \\
& \left.+C_{A}^{2} T_{f}\left(1892352 C_{A}+2973696 C_{f}\right) \frac{d_{f}^{a b c d} d_{f^{\prime}}^{a b c d}}{d_{A}}\right] \\
& A_{2}^{(f)}=T_{f}^{2} T_{f^{\prime}}^{2}\left[350976 C_{A}^{2}\left(C_{f}-C_{f^{\prime}}\right)^{2}+C_{A}\left(-94464 C_{f}^{3}-2304 C_{f}^{2} C_{f^{\prime}}+288000 C_{f} C_{f^{\prime}}^{2}-191232 C_{f^{\prime}}^{3}\right)\right. \\
& \left.+225792 C_{f}^{4}-370944 C_{f}^{3} C_{f^{\prime}}+119808 C_{f}^{2} C_{f^{\prime}}^{2}-29952 C_{f} C_{f^{\prime}}^{3}+55296 C_{f^{\prime}}^{4}\right] \\
& +2{ }^{16} T_{f} T_{f^{\prime}}\left(C_{f}-C_{f^{\prime}}\right)\left(T_{f^{\prime}} \frac{d_{f}^{a b c d} d_{A}^{a b c d}}{d_{A}}-T_{f} \frac{d_{f^{\prime}}^{a b c d} d_{A}^{a b c d}}{d_{A}}\right)+C_{A} T_{f^{\prime}}^{2}\left(-157696 C_{A}+495616 C_{f^{\prime}}-743424 C_{f}\right) \frac{d_{f}^{a b c d} d_{f}^{a b c d}}{d_{A}} \\
& +C_{A} T_{f} T_{f^{\prime}}\left(315392 C_{A}+991232 C_{f}-495616 C_{f^{\prime}}\right) \frac{d_{f}^{a b c d} d_{f^{\prime}}^{a b c d}}{d_{A}}+C_{A} T_{f}^{2}\left(-157696 C_{A}-247808 C_{f}\right) \frac{d_{f^{\prime}}^{a b c d} d_{f^{\prime}}^{a b c d}}{d_{A}} \\
& +\zeta_{3}\left[638976 T_{f} T_{f^{\prime}}\left(C_{f}-C_{f^{\prime}}\right)\left(T_{f} \frac{d_{f^{\prime}}^{a b c d} d_{A}^{a b c d}}{d_{A}}-T_{f^{\prime}} \frac{d_{f}^{a b c d} d_{A}^{a b c d}}{d_{A}}\right)\right. \\
& +C_{A} T_{f^{\prime}}^{2}\left(344064 C_{A}+1622016 C_{f}-1081344 C_{f^{\prime}}\right) \frac{d_{f}^{a b c d} d_{f}^{a b c d}}{d_{A}} \\
& \left.\times C_{A} T_{f} T_{f^{\prime}}\left(-688128 C_{A}-2162688 C_{f}+1081344 C_{f^{\prime}}\right) \frac{d_{f}^{a b c d} d_{f^{\prime}}^{a b c d}}{d_{A}}+C_{A} T_{f}^{2}\left(344064 C_{A}+540672 C_{f}\right) \frac{d_{f^{\prime}}^{a b c d} d_{f^{\prime}}^{a b c d}}{d_{A}}\right]
\end{aligned}
$$

and

$$
A_{3}^{(f)}=2^{13} T_{f^{\prime}}\left(C_{f}-C_{f^{\prime}}\right)\left(11-24 \zeta_{3}\right)\left(T_{f^{\prime}}^{2} \frac{d_{f}^{a b c d} d_{f}^{a b c d}}{d_{A}}-2 T_{f} T_{f^{\prime}} \frac{d_{f}^{a b c d} d_{f^{\prime}}^{a b c d}}{d_{A}}+T_{f}^{2} \frac{d_{f^{\prime}}^{a b c d} d_{f^{\prime}}^{a b c d}}{d_{A}}\right)
$$

The coefficients $\kappa_{j}^{\left(f^{\prime}\right)}$ are obtained from these $\kappa_{j}^{(f)}$ by interchanging $f$ and $f^{\prime}$ in all expressions. For example,

$$
\begin{gathered}
\mathcal{D}_{f^{\prime}}=C_{A}\left(7 C_{A}+11 C_{f^{\prime}}\right)+4 N_{f} T_{f}\left(C_{f}-C_{f^{\prime}}\right), \\
\kappa_{1}^{\left(f^{\prime}\right)}=\frac{8 C_{f^{\prime}} T_{f^{\prime}}}{\mathcal{D}_{f^{\prime}}},
\end{gathered}
$$

and so forth for the other expressions. 
An important result that we found in our previous work [12-16] was that for a theory with a single representation, $\kappa_{1}^{(f)}$ and $\kappa_{2}^{(f)}$ are manifestly positive, and for all of the specific gauge groups and fermion representations that we considered, $\kappa_{3}^{(f)}$ and $\kappa_{4}^{(f)}$ are also positive. This property implied several monotonicity relations for our calculation of $\gamma_{\bar{\psi} \psi}$ to maximal power $\Delta_{f}^{p}$, denoted $\gamma_{\bar{\psi} \psi, \Delta_{f}^{p}}$, namely that (i) for fixed $p, \gamma_{\bar{\psi} \psi, \Delta_{f}^{p}}$ is a monotonically increasing function of $\Delta_{f}$, i.e., a monotonically increasing function of decreasing $N_{f}$, and (ii) for fixed $N_{f}, \gamma_{\bar{\psi} \psi, \Delta_{f}^{p}}$ is a monotonically increasing function of the maximal power $p$.

A basic question that we may ask concerning these results is how a coefficient $\kappa^{(f)}$ changes as one goes from the single-representation theory with $N_{f}^{\prime}=0$ to theories with an increasing number $N_{f^{\prime}}$ of fermions in a different representation, and vice versa for the dependence of $\kappa^{\left(f^{\prime}\right)}$ on $N_{f}$. For the purpose of this discussion, we recall that, by convention, we take $f$ to be the fermion in the representation with a smaller dimension. In the cases with which we deal, this also means that $C_{f}<C_{f^{\prime}}$. The question is readily answered in the case of $\kappa_{1}^{(f)}$ and $\kappa_{1}^{\left(f^{\prime}\right)}$. As a lemma, we observe that $\mathcal{D}_{f}$ is a monotonically increasing function of $N_{f^{\prime}}$, while $\mathcal{D}_{f^{\prime}}$ is a monotonically decreasing function of $N_{f}$. Hence, $\kappa_{1}^{(f)}$ is a monotonically decreasing function of $N_{f^{\prime}}$, while $\kappa_{1}^{\left(f^{\prime}\right)}$ is a monotonically increasing function of $N_{f}$. The dependence of $\kappa_{j}^{(f)}$ on $N_{f^{\prime}}$ and of $\kappa_{j}^{\left(f^{\prime}\right)}$ on $N_{f}$ for indices $j=2,3$ will be analyzed below for particular theories.

Concerning the question of the positivity of $\kappa_{j}^{(f)}$ and $\kappa_{j}^{\left(f^{\prime}\right)}$, in a theory with fermions in multiple different representations, there are terms of both signs in the expressions for the coefficients $\kappa_{j}^{(f)}$. Nevertheless, anticipating our results below, in the specific FA theories that we have studied in detail, both $\kappa_{j}^{(F)}$ and $\kappa_{j}^{(\text {Adj) }}$ are positive for all of the orders that we have calculated, namely $j=1,2,3$.

In our earlier work [11-17] on scheme-independent series calculations for theories with a $N_{f}$ fermions transforming according to a single type of representation, we carried out detailed studies of the reliability of these expansions using a variety of methods. One of the simplest procedures is to analyze the fractional change in a quantity, calculated to a given order $O\left(\Delta_{f}^{p}\right)$, as one increases the maximal power $p$ of the expansion. Here we shall apply this method in our illustrative theory discussed in the next section.

\section{ANOMALOUS DIMENSIONS IN A THEORY WITH FERMIONS IN THE FUNDAMENTAL AND ADJOINT REPRESENTATIONS OF SU $\left(N_{c}\right)$}

In this section we discuss our scheme-independent calculations of $\gamma_{\bar{\psi} \psi, \text { IR }}$ and $\gamma_{\bar{\chi} \chi}$,IR for the illustrative case of a theory with gauge group $\mathrm{SU}\left(N_{c}\right)$ containing $N_{f} \equiv N_{F}$ fermions in the fundamental representation and $N_{f^{\prime}} \equiv N_{\text {Adj }}$ fermions in the adjoint representation. As before, we call this the FA theory. In this case, the denominator factor $\mathcal{D}_{f}$ takes the form

FA theory: $\mathcal{D}_{f}=\frac{1}{2}\left[25 N_{c}^{2}-11+4 N_{A d j}\left(N_{c}^{2}+1\right)\right]$.

We have given the values of $\left(N_{F}, N_{A d j}\right)$ in Table I for the region $\mathcal{R}_{\text {IRZ }}$. For the first-order coefficients we calculate

$$
\kappa_{1}^{(F)}=\frac{4\left(N_{c}^{2}-1\right)}{N_{c}\left[25 N_{c}^{2}-11+4 N_{A d j}\left(N_{c}^{2}+1\right)\right]}
$$

and

$$
\kappa_{1}^{(A d j)}=\frac{8 N_{c}^{3}}{18 N_{c}^{3}-N_{F}\left(N_{c}^{2}+1\right)} .
$$

If $N_{A d j}=0$, then the coefficient $\kappa_{1}^{(F)}$ reduces to the expression $4\left(N_{c}^{2}-1\right) /\left[N_{c}\left(25 N_{c}^{2}-11\right)\right]$, as given in Eq. (6.8) of our earlier work [13]. Similarly, if $N_{F}=0$, then $\kappa_{1}^{(A d j)}$ reduces to the value 4/9, as given in Eq. (6.18) of [13].

For the second-order coefficients, we find

$$
\kappa_{2}^{(F)}=\frac{4\left(N_{c}^{2}-1\right)\left[\left(9 N_{c}^{2}-2\right)\left(49 N_{c}^{2}-44\right)+8 N_{A d j}\left(N_{c}^{2}+1\right)\left(15 N_{c}^{2}-4\right)\right]}{3 N_{c}^{2}\left[25 N_{c}^{2}-11+4 N_{A d j}\left(N_{c}^{2}+1\right)\right]^{3}}
$$

and

$\kappa_{2}^{(A d j)}=\frac{4 N_{c}^{4}\left[1023 N_{c}^{5}-2 N_{F}\left(N_{c}^{2}+1\right)\left(37 N_{c}^{2}-1\right)\right]}{3\left[18 N_{c}^{3}-N_{F}\left(N_{c}^{2}+1\right)\right]^{3}}$.

If $N_{A d j}=0$, then $\kappa_{2}^{(F)}$ reduces to the expression given in Eq. (6.9) of [13], and if $N_{F}=0$, then $\kappa_{2}^{(A d j)}$ reduces to the value $341 / 1458=341 /\left(2 \cdot 3^{6}\right)$ as given in Eq. (6.19) of [13].

Our results for the third-order coefficients are as follows:

$$
\kappa_{3}^{(F)}=\frac{2^{3}\left(N_{c}^{2}-1\right)\left[\kappa_{3,0}^{(F)}+\kappa_{3,1}^{(F)} N_{A d j}+\kappa_{3,2}^{(F)} N_{A d j}^{2}+\kappa_{3,3}^{(F)} N_{A d j}^{3}\right]}{3^{3} N_{c}^{3}\left[25 N_{c}^{2}-11+4 N_{A d j}\left(N_{c}^{2}+1\right)\right]^{5}},
$$


where

$$
\begin{aligned}
\kappa_{3,0}^{(F)}= & 274243 N_{c}^{8}-455426 N_{c}^{6}-114080 N_{c}^{4}+47344 N_{c}^{2} \\
& +35574-2^{7} \cdot 33 \zeta_{3} N_{c}^{2}\left(4 N_{c}^{2}-11\right)\left(25 N_{c}^{2}-11\right)
\end{aligned}
$$

$$
\begin{aligned}
\kappa_{3,1}^{(F)}= & 135848 N_{c}^{8}-215832 N_{c}^{6}+291424 N_{c}^{4}-189168 N_{c}^{2} \\
& -25872-2^{9} \cdot 3^{2} \zeta_{3} N_{c}^{2}\left(73 N_{c}^{4}+132 N_{c}^{2}-121\right)
\end{aligned}
$$

$$
\begin{aligned}
\kappa_{3,2}^{(F)}= & 2^{5}\left(N_{c}^{2}+1\right)\left[689 N_{c}^{6}-2651 N_{c}^{4}+2775 N_{c}^{2}\right. \\
& \left.+147+2^{6} \cdot 3^{2} \zeta_{3} N_{c}^{2}\left(6 N_{c}^{2}-11\right)\right]
\end{aligned}
$$

and

$$
\kappa_{3,3}^{(F)}=2^{10} N_{c}^{2}\left(N_{c}^{2}+1\right)^{2}\left(-11+24 \zeta_{3}\right) .
$$

Further,

$$
\kappa_{3}^{(A d j)}=\frac{4 N_{c}^{5}\left[\kappa_{3,0}^{(A d j)}+\kappa_{3,1}^{(A d j)} N_{F}+\kappa_{3,2}^{(A d j)} N_{F}^{2}+\kappa_{3,3}^{(A d j)} N_{F}^{3}\right]}{3^{3}\left[18 N_{c}^{3}-N_{F}\left(N_{c}^{2}+1\right)\right]^{5}},
$$

where

$$
\begin{aligned}
& \kappa_{3,0}^{(A d j)}=3^{3} N_{c}^{8}\left(61873 N_{c}^{2}-42624\right) \\
\kappa_{3,1}^{(A d j)}= & -36 N_{c}^{3}\left(6728 N_{c}^{6}-5857 N_{c}^{4}-1247 N_{c}^{2}\right. \\
& \left.+138+11520 \zeta_{3} N_{c}^{4}\right) \\
\kappa_{3,2}^{(A d j)}= & 2^{5}\left(N_{c}^{2}+1\right)\left(287 N_{c}^{6}-1187 N_{c}^{4}+27 N_{c}^{2}\right. \\
& \left.+9+2448 \zeta_{3} N_{c}^{4}\right)
\end{aligned}
$$

and

$$
\kappa_{3,3}^{(A d j)}=-2^{7} N_{c}\left(N_{c}^{2}+1\right)^{2}\left(-11+24 \zeta_{3}\right) .
$$

If $N_{A d j}=0$, then the coefficient $\kappa_{3}^{(F)}$ reduces to the expression in Eq. (6.10) of our earlier work [13], while if $N_{F}=0$, then $\kappa_{3}^{(A d j)}$ reduces to Eq. (6.20) of [13]. The agreement of these reductions of $\kappa_{j}^{(F)}$ for $N_{A d j}=0$ and of $\kappa_{j}^{(A d j)}$ for $N_{F}=0$ with our earlier calculations in [13] for $j=1,2,3$ serves as a check on our present results. As was discussed in $[13,15]$, these coefficients have the leading large- $N_{c}$ dependence

$$
\kappa_{j}^{(F)} \sim N_{c}^{-j} \quad \text { as } N_{c} \rightarrow \infty
$$

TABLE II. Values of the coefficients $\kappa_{j}^{(F)}, j=1,2,3$, for the scheme-independent expansion of the anomalous dimension $\gamma_{\bar{\psi} \mu, \text { IR }}$ in an SU(3) gauge theory with fermions in the fundamental and adjoint representations, as functions of $N_{A d j}$. Half-integral values of $N_{A d j}$ correspond to $2 N_{A d j}$ copies of Majorana fermions in the adjoint representation. The notation $a$ e-n means $a \times 10^{-n}$.

\begin{tabular}{lccl}
\hline \hline$N_{\text {Adj }}$ & $\kappa_{1}^{(F)}$ & $\kappa_{2}^{(F)}$ & \multicolumn{1}{c}{$\kappa_{3}^{(F)}$} \\
\hline 0 & $4.98 \mathrm{e}-2$ & $3.79 \mathrm{e}-3$ & $2.37 \mathrm{e}-4$ \\
$\frac{1}{2}$ & $4.56 \mathrm{e}-2$ & $3.39 \mathrm{e}-3$ & $1.835 \mathrm{e}-4$ \\
1 & $4.20 \mathrm{e}-2$ & $3.03 \mathrm{e}-3$ & $1.51 \mathrm{e}-4$ \\
$\frac{3}{2}$ & $3.89 \mathrm{e}-2$ & $2.71 \mathrm{e}-3$ & $1.31 \mathrm{e}-4$ \\
2 & $3.63 \mathrm{e}-2$ & $2.44 \mathrm{e}-3$ & $1.16 \mathrm{e}-4$ \\
\hline \hline
\end{tabular}

and

$$
\kappa_{j}^{(A d j)} \sim N_{c}^{0} \quad \text { as } N_{c} \rightarrow \infty .
$$

As specific examples of these FA theories, we consider the following sets of SU(3) gauge theories in $\mathcal{R}_{\mathrm{IRZ}}$ with the indicated fermion content:

$$
\begin{aligned}
\left(N_{F}, N_{\text {Adj }}\right)= & \left(8, \frac{1}{2}\right), \quad(8,1), \quad(10,0), \quad\left(10, \frac{1}{2}\right), \\
& (10,1), \quad(12,0), \quad\left(12, \frac{1}{2}\right)
\end{aligned}
$$

The respective positions of these theories in the regions $\mathcal{R}_{\text {IRZ }}$ and $\mathcal{R}_{\mathrm{NACP}}$ can be ascertained by referring to Table I. The corresponding values of the coefficients $\kappa_{j}^{(F)}$ with $j=1,2,3$, as functions of $N_{A d j}$, are listed in Table II, and the values of $\kappa_{j}^{(A d j)}$ with $j=1,2,3$, as functions of $N_{F}$, are listed in Table III.

We observe that all of these coefficients are positive, and so the generalizations of the monotonicity relations that we found in our earlier work for the theory with fermions in a single representation also hold for this FA theory, namely (i) for fixed $N_{A d j}, \gamma_{\bar{\psi} \psi, \text { IR }}$ is a monotonically increasing function of $\Delta_{F}$, i.e., a monotonically increasing function of decreasing $N_{F}$; (ii) for fixed $N_{F}, \gamma_{\bar{\chi} \chi}$,IR is a monotonically increasing function of $\Delta_{A d j}$, i.e., a monotonically increasing function of decreasing $N_{A d j}$; (iii) for fixed $N_{f^{\prime}}, \gamma_{\bar{\psi} \mu, \mathrm{IR}, \Delta_{F}^{p}}$

TABLE III. Values of the coefficients $\kappa_{j}^{(\operatorname{Adj})}, j=1,2,3$, for the scheme-independent expansion of the anomalous dimension $\gamma_{\bar{\chi} \chi, I R}$ in an SU(3) gauge theory with fermions in the fundamental and adjoint representations, for illustrative values of $N_{F}$.

\begin{tabular}{llll}
\hline \hline$N_{F}$ & $\kappa_{1}^{(\text {Adj })}$ & $\kappa_{2}^{(\text {Adj })}$ & $\kappa_{3}^{(\text {Adj })}$ \\
\hline 0 & 0.444 & 0.234 & 0.121 \\
4 & 0.484 & 0.270 & 0.145 \\
8 & 0.532 & 0.315 & 0.179 \\
10 & 0.560 & 0.342 & 0.201 \\
12 & 0.590 & 0.372 & 0.227 \\
\hline \hline
\end{tabular}


is a monotonically increasing function of $p$; and (iv) for fixed $N_{f}, \gamma_{\bar{\chi} \chi, \mathrm{IR}, \Delta_{A d j}^{p}}$ is a monotonically increasing function of $p$.

Separately, we also note a generalization of the monotonicity relation that we proved for $\kappa_{1}^{(F)}$ and proved for $\kappa_{1}^{(A d j)}$, namely that for these FA theories, the $\kappa_{j}^{(F)}$ coefficients with $j=1,2,3$ are monotonically decreasing functions of $N_{A d j}$, and the $\kappa_{j}^{(A d j)}$ coefficients with $j=1,2,3$ are monotonically increasing functions of $N_{F}$.

Having calculated these coefficients $\kappa_{j}^{(F)}$ and $\kappa_{j}^{(\text {Adj) }}$ with $j=1,2,3$ for this FA theory, we next proceed to substitute them in the general scheme-independent expansions (1.6) for $f=F$ and the analogue for $f^{\prime}=A d j$. Explicitly, with $f=\psi$ and $f^{\prime}=\chi$,

$$
\gamma_{\bar{\psi} \psi, \mathrm{IR}}=\sum_{j=1}^{\infty} \kappa_{j}^{(F)} \Delta_{F}^{j}
$$

and

$$
\gamma_{\bar{\chi} \chi, \mathrm{IR}}=\sum_{j=1}^{\infty} \kappa_{j}^{(A d j)} \Delta_{A d j}^{j},
$$

where

$$
\Delta_{F}=\left(N_{F, u}-N_{F}\right)
$$

with

$$
N_{F, u}=\frac{N_{c}\left(11-4 N_{A d j}\right)}{2}
$$

and

$$
\Delta_{A d j}=\left(N_{A d j, u}-N_{A d j}\right)
$$

with

$$
N_{A d j, u}=\frac{11}{4}-\frac{N_{F}}{2 N_{c}} .
$$

For reference, we list the values of $N_{F, u}$ and $N_{A d j, u}$ from Eqs. (4.22) and (4.24) for these $\left(N_{F}, N_{A d j}\right)$ FA SU(3) theories in Table IV.

In Table $\mathrm{V}$ we list the values of $\gamma_{\bar{\psi} \psi, \text { IR }}$ calculated to $O\left(\Delta_{F}^{p}\right)$ for $p=1,2,3$, denoted as $\gamma_{\bar{\psi} \psi, \mathrm{IR}, \Delta_{F}^{p}}$. Similarly, in Table VI we list the values of $\gamma_{\bar{\chi} \chi, \text { IR }}$ calculated to $O\left(\Delta_{A d j}^{p}\right)$ for $p=1,2,3$, denoted as $\gamma_{\bar{\chi} \chi, I R, \Delta_{A d j}^{p}}$. The monotonicity relations noted above are evident in these tables. From an examination of the fractional changes in the anomalous dimensions as one increases the order of calculation, one may infer that these scheme-independent expansions should be reasonably reliable. For example, in the SU(3) FA theory with $\left(N_{F}, N_{A d j}\right)=(12,1 / 2)$ theory, the fractional change in the $\gamma_{\bar{\psi} \psi, \mathrm{IR}}$ anomalous dimension is
TABLE IV. Values of $N_{F, u}$ from Eq. (2.4) and $N_{A d j, u}$ from Eq. (2.5) (formally generalized to non-negative real numbers) for the illustrative SU(3) theories with $N_{F}$ fermions in the fundamental representation and $N_{A d j}$ fermions in the adjoint representation. Half-integral values of $N_{A d j}$ refer to theories with $2 N_{A d j}$ Majorana fermions in the adjoint representation.

\begin{tabular}{lcc}
\hline \hline$\left(N_{F}, N_{A d j}\right)$ & $N_{F, u}$ & $N_{A d j, u}$ \\
\hline$(8,1 / 2)$ & $27 / 2$ & $17 / 12$ \\
$(8,1)$ & $21 / 2$ & $17 / 12$ \\
$(10,0)$ & $33 / 2$ & $13 / 12$ \\
$(10,1 / 2)$ & $27 / 2$ & $13 / 12$ \\
$(10,1)$ & $21 / 2$ & $13 / 12$ \\
$(12,0)$ & $33 / 2$ & $3 / 4$ \\
$(12,1 / 2)$ & $27 / 2$ & $3 / 4$ \\
\hline \hline
\end{tabular}

TABLE V. Values of the anomalous dimension $\gamma_{\bar{\psi} \psi, \mathrm{IR}, \Delta_{F}^{p}}$, calculated to order $p=1,2,3$ and evaluated at the IR fixed point in an SU(3) gauge theory with $N_{F}$ fermions in the fundamental $(F)$ representation and $N_{A d j}$ fermions in the adjoint ( $A d j$ ) representation. Here, $\psi$ is the fermion in the $F$ representation.

\begin{tabular}{lccc}
\hline \hline$\left(N_{F}, N_{A d j}\right)$ & $\gamma_{\bar{\psi} \psi, \mathrm{IR}, \Delta_{F}}$ & $\gamma_{\bar{\mu} \mu, \mathrm{IR}, \Delta_{F}^{2}}$ & $\gamma_{\bar{\mu} \bar{\mu}, \mathrm{IR}, \Delta_{F}^{3}}$ \\
\hline$(8,1 / 2)$ & 0.251 & 0.353 & 0.384 \\
$(8,1)$ & 0.105 & 0.124 & 0.126 \\
$(10,0)$ & 0.324 & 0.484 & 0.549 \\
$(10,1 / 2)$ & 0.159 & 0.201 & 0.209 \\
$(10,1)$ & 0.0210 & 0.0218 & 0.0218 \\
$(12,0)$ & 0.224 & 0.301 & 0.323 \\
$(12,1 / 2)$ & 0.0684 & 0.0760 & 0.0766 \\
\hline \hline
\end{tabular}

TABLE VI. Values of the anomalous dimension $\gamma_{\bar{\chi} \chi, I R, \Delta_{A d j}^{p}}$, calculated to order $p=1,2,3$ and evaluated at the IR fixed point in an SU(3) gauge theory with $N_{F}$ fermions in the fundamental $(F)$ representation and $N_{A d j}$ fermions in the adjoint (Adj) representation. Here, $\chi$ is the fermion in the $A d j$ representation.

\begin{tabular}{lccc}
\hline \hline$\left(N_{F}, N_{A d j}\right)$ & $\gamma_{\bar{\chi} \chi, \mathrm{IR}, \Delta_{A d j}}$ & $\gamma_{\bar{\chi} \chi, \mathrm{IR}, \Delta_{A d j}^{2}}$ & $\gamma_{\bar{\chi} \chi, \mathrm{IR}, \Delta_{A d j}^{3}}$ \\
\hline$(8,1 / 2)$ & 0.488 & 0.753 & 0.891 \\
$(8,1)$ & 0.222 & 0.276 & 0.289 \\
$(10,1 / 2)$ & 0.326 & 0.443 & 0.483 \\
$(10,1)$ & 0.0466 & 0.0490 & 0.0491 \\
$(12,1 / 2)$ & 0.1475 & 0.171 & 0.174 \\
\hline \hline
\end{tabular}

$$
\begin{aligned}
& \mathrm{SU}(3),\left(N_{F}, N_{A d j}\right)=(12,1 / 2) \\
& \Rightarrow \frac{\gamma_{\bar{\psi} \psi}, \mathrm{IR}, \Delta_{F}^{3}}{}-\gamma_{\bar{\psi} \psi, \mathrm{IR}, \Delta_{F}^{2}}=0.81 \times 10^{-2} . \\
& \gamma_{\bar{\psi} \psi, \mathrm{IR}, \Delta_{F}^{2}}
\end{aligned}
$$

In the $\mathrm{SU}(3)$ FA theory with $\left(N_{F}, N_{A d j}\right)=(10,1)$ theory, the fractional change in $\gamma_{\bar{\psi} \psi, \text { IR }}$ is even smaller: 


$$
\begin{aligned}
\mathrm{SU}(3),\left(N_{F}, N_{A d j}\right) & =(10,1) \\
\Rightarrow \frac{\gamma_{\bar{\psi} \psi, \mathrm{IR}, \Delta_{F}^{3}}-\gamma_{\bar{\psi} \psi, \mathrm{IR}, \Delta_{F}^{2}}}{\gamma_{\bar{\psi} \psi, \mathrm{IR}, \Delta_{F}^{2}}} & =0.87 \times 10^{-3},
\end{aligned}
$$

yielding identical entries listed to three significant figures in Table V. Similar comments apply to the calculations of $\gamma_{\bar{\chi} \chi}, \mathrm{IR}, \Delta_{A d j}^{p}$.

\section{SCHEME-INDEPENDENT CALCULATION OF $\boldsymbol{\beta}_{\mathrm{IR}}^{\prime}$}

In this section we return to the general asymptotically free gauge theory with gauge group $G$ containing $N_{f}$ and $N_{f^{\prime}}$ fermions in the respective representations $R$ and $R^{\prime}$ and present our calculations of the coefficients $d_{j}$ and $\tilde{d}_{j}$ in the scheme-independent expansions of the derivative of the beta function evaluated at the IR fixed point, $\beta_{\mathrm{IR}}^{\prime}$, in powers of $\Delta_{f}$ in Eqs. (1.8) and in powers of $\Delta_{f^{\prime}}$ in Eq. (1.9), respectively. As before in this paper, this IR fixed point is taken to be in the non-Abelian Coulomb phase. Part of the physical interest in the quantity $\beta_{\mathrm{IR}}^{\prime}$ stems from the fact that, owing to the trace anomaly relation [42], it is equivalent to the anomalous dimension of the field-strength tensor term $\operatorname{Tr}\left(F_{\mu \nu}^{a} F^{a \mu \nu}\right)$ in the Lagrangian [13,43]. As noted above, generalizing our result for the single-representation case, $d_{1}=\tilde{d}_{1}=0$ for arbitrary $G, R$, and $R^{\prime}$.

For the higher coefficients we find

$$
\begin{gathered}
d_{2}=\frac{2^{5} T_{f}^{2}}{3^{2} \mathcal{D}_{f}} \\
d_{3}=\frac{2^{7} T_{f}^{3}\left(5 C_{A}+3 C_{f}\right)}{3^{3} \mathcal{D}_{f}^{2}}
\end{gathered}
$$

and

$d_{4}=-\frac{2^{3} T_{f}^{2}}{3^{6} \mathcal{D}_{f}^{5}}\left[B_{0}^{(f)}+B_{1}^{(f)} N_{f^{\prime}}+B_{2}^{(f)} N_{f^{\prime}}^{2}+B_{3}^{(f)} N_{f^{\prime}}^{3}\right]$,

where we explicitly indicate the dependence on $f$ in the $B_{s}^{(f)}, s=0,1,2,3$. [We extract a minus sign in Eq. (5.3) to maintain the same notation as in our earlier works $[13,15]$, where we found that in the case of fermions in a single representation $R=F, d_{4}$ is negative.] As was the case with $A_{0}^{(f)}$ in $\kappa_{3}^{(f)}$, the $B_{0}^{(f)}$ term in $d_{4}$ is independent of $N_{f^{\prime}}$ and hence, taking into account the difference in the prefactor, it is equal to $C_{A}$ times the terms in the square bracket of Eq. (5.11) in our earlier Ref. [13] or equivalently, Eq. (4.8) of our Ref. [15]. We have

$$
\begin{aligned}
B_{0}^{(f)}= & C_{A}\left[-3 C_{A} T_{f}^{2}\left(137445 C_{A}^{4}+103600 C_{A}^{3} C_{f}+72616 C_{A}^{2} C_{f}^{2}+951808 C_{A} C_{f}^{3}-63888 C_{f}^{4}\right)\right. \\
& +2^{8} D\left(-20 T_{f}^{2} \frac{d_{A}^{a b c d} d_{A}^{a b c d}}{d_{A}}+352 C_{A} T_{f} \frac{d_{f}^{a b c d} d_{A}^{a b c d}}{d_{A}}-1331 C_{A}^{2} \frac{d_{f}^{a b c d} d_{f}^{a b c d}}{d_{A}}\right) \\
& \left.+8448 D \zeta_{3}\left\{C_{A}^{2} T_{f}^{2}\left(21 C_{A}^{2}+12 C_{A} C_{f}-33 C_{f}^{2}\right)+16 T_{f}^{2} \frac{d_{A}^{a b c d} d_{A}^{a b c d}}{d_{A}}-104 C_{A} T_{f} \frac{d_{f}^{a b c d} d_{A}^{a b c d}}{d_{A}}+88 C_{A}^{2} \frac{d_{f}^{a b c d} d_{f}^{a b c d}}{d_{A}}\right\}\right] .
\end{aligned}
$$

For the $B_{j}^{(f)}$ with $j=1,2,3$, we calculate

$$
\begin{aligned}
B_{1}^{(f)}= & 194880 C_{A}^{4} T_{f}^{2} T_{f^{\prime}}\left(C_{f^{\prime}}-C_{f}\right)+C_{A}^{3} T_{f}^{2} T_{f^{\prime}}\left(-1854816 C_{f}^{2}+2715648 C_{f} C_{f^{\prime}}-860832 C_{f^{\prime}}^{2}\right) \\
& +C_{A}^{2} T_{f}^{2} T_{f^{\prime}}\left(903168 C_{f}^{3}+153216 C_{f}^{2} C_{f^{\prime}}-1241856 C_{f} C_{f^{\prime}}^{2}+185472 C_{f^{\prime}}^{3}\right) \\
& +C_{A} T_{f}^{2} T_{f^{\prime}} C_{f}\left(-139392 C_{f}^{3}-164736 C_{f}^{2} C_{f^{\prime}}+12672 C_{f} C_{f^{\prime}}^{2}+291456 C_{f^{\prime}}^{3}\right) \\
& +C_{A}^{2} T_{f}^{2} T_{f^{\prime}}\left(C_{f}-C_{f^{\prime}}\right) \zeta_{3}\left(-967680 C_{A}^{2}+608256 C_{A} C_{f}+3345408 C_{f}^{2}\right) \\
& +T_{f}^{2} T_{f^{\prime}}\left(C_{f}-C_{f^{\prime}}\right)\left(20480-540672 \zeta_{3}\right) \frac{d_{A}^{a b c d} d_{A}^{a b c d}}{d_{A}} \\
& +C_{A} T_{f} T_{f^{\prime}}\left[-229376 C_{A}-720896 C_{f}+360448 C_{f^{\prime}}+\zeta_{3}\left(2236416 C_{A}+7028736 C_{f}-3514368 C_{f^{\prime}}\right)\right] \frac{d_{f}^{a b c d} d_{A}^{a b c d}}{d_{A}} \\
& +C_{A} T_{f}^{2}\left[229376 C_{A}+360448 C_{f}+\zeta_{3}\left(-2236416 C_{A}-3514368 C_{f}\right)\right] \frac{d_{f^{\prime}}^{a b c d} d_{A}^{a b c d}}{d_{A}} \\
& +C_{A}^{2} T_{f^{\prime}}\left[1734656 C_{A}+4088832 C_{f}-1362944 C_{f^{\prime}}+\zeta_{3}\left(-3784704 C_{A}-8921088 C_{f}+2973696 C_{f^{\prime}}\right)\right] \frac{d_{f}^{a b c d} d_{f}^{a b c d}}{d_{A}} \\
& +C_{A}^{2} T_{f}\left[C_{A}\left(-1734656+3784704 \zeta_{3}\right)+C_{f}\left(-2725888+5947392 \zeta_{3}\right)\right] \frac{d_{f}^{a b c d} d_{f^{\prime}}^{a b c d}}{d_{A}}
\end{aligned}
$$




$$
\begin{aligned}
& B_{2}^{(f)}=T_{f}^{2} T_{f^{\prime}}^{2}\left[669696 C_{A}^{2}\left(C_{f}-C_{f^{\prime}}\right)^{2}+C_{A}\left(437760 C_{f}^{3}-1327104 C_{f}^{2} C_{f^{\prime}}+1340928 C_{f} C_{f^{\prime}}^{2}-451584 C_{f^{\prime}}^{3}\right)\right. \\
& +25344 C_{f}^{4}+59904 C_{f}^{3} C_{f^{\prime}}-87552 C_{f}^{2} C_{f^{\prime}}^{2}-105984 C_{f} C_{f^{\prime}}^{3}+108288 C_{f^{\prime}}^{4} \\
& \left.+C_{A}\left(C_{f}-C_{f^{\prime}}\right)^{2} \zeta_{3}\left(-110592 C_{A}-1216512 C_{f}\right)\right] \\
& +T_{f} T_{f^{\prime}}\left(C_{f}-C_{f^{\prime}}\right)\left(131072-1277952 \zeta_{3}\right)\left[T_{f^{\prime}} \frac{d_{f}^{a b c d} d_{A}^{a b c d}}{d_{A}}-T_{f} \frac{d_{f^{\prime}}^{a b c d} d_{A}^{a b c d}}{d_{A}}\right] \\
& +C_{A} T_{f^{\prime}}^{2}\left[-315392 C_{A}-1486848 C_{f}+991232 C_{f^{\prime}}+\zeta_{3}\left(688128 C_{A}+3244032 C_{f}-2162688 C_{f^{\prime}}\right)\right] \frac{d_{f}^{a b c d} d_{f}^{a b c d}}{d_{A}} \\
& +C_{A} T_{f} T_{f^{\prime}}\left[630784 C_{A}+1982464 C_{f}-991232 C_{f^{\prime}}+\zeta_{3}\left(-1376256 C_{A}-4325376 C_{f}+2162688 C_{f^{\prime}}\right)\right] \frac{d_{f}^{a b c d} d_{f^{\prime}}^{a b c d}}{d_{A}} \\
& +C_{A} T_{f}^{2}\left[-315392 C_{A}-495616 C_{f}+\zeta_{3}\left(688128 C_{A}+1081344 C_{f}\right)\right] \frac{d_{f^{\prime}}^{a b c d} d_{f^{\prime}}^{a b c d}}{d_{A}}
\end{aligned}
$$

and

$$
\begin{aligned}
B_{3}^{(f)}= & T_{f^{\prime}}\left(C_{f}-C_{f^{\prime}}\right)\left[2^{11} \cdot 3 T_{f}^{2} T_{f^{\prime}}^{2}\left(C_{f}-C_{f^{\prime}}\right)^{2}\left(-23+24 \zeta_{3}\right)\right. \\
& \left.+2^{14}\left(11-24 \zeta_{3}\right)\left(T_{f^{\prime}}^{2} \frac{d_{f}^{a b c d} d_{f}^{a b c d}}{d_{A}}-2 T_{f} T_{f^{\prime}} \frac{d_{f}^{a b c d} d_{f^{\prime}}^{a b c d}}{d_{A}}+T_{f}^{2} \frac{d_{f^{\prime}}^{a b c d} d_{f^{\prime}}^{a b c d}}{d_{A}}\right)\right]
\end{aligned}
$$

In passing, we note that $B_{3}^{(f)}$ has the same prefactor as $A_{3}^{(f)}$ in Eq. (3.10), namely $T_{f^{\prime}}\left(C_{f}-C_{f^{\prime}}\right)$.

The corresponding coefficients for the expansion (1.9) are obtained from these by interchanging $f$ and $f^{\prime}$. Thus, for example,

TABLE VII. Values of the coefficients $d_{j}, j=2,3,4$, for the scheme-independent expansion of $\beta_{\mathrm{IR}}^{\prime}$, Eq. (6.1), in an SU(3) gauge theory with fermions in the fundamental and adjoint representations, as functions of $N_{\text {Adj }}$. Half-integral values of $N_{A d j}$ correspond to $2 N_{A d j}$ copies of Majorana fermions in the adjoint representation. The notation ae-n means $a \times 10^{-n}$.

\begin{tabular}{lcll}
\hline \hline$N_{\text {Adj }}$ & \multicolumn{1}{c}{$d_{2}$} & \multicolumn{1}{c}{$d_{3}$} & \multicolumn{1}{c}{$d_{4}$} \\
\hline 0 & $0.831 \mathrm{e}-2$ & $0.983 \mathrm{e}-3$ & $-0.463 \mathrm{e}-4$ \\
$1 / 2$ & $0.760 \mathrm{e}-2$ & $0.8225 \mathrm{e}-3$ & $-2.44 \mathrm{e}-5$ \\
1 & $0.700 \mathrm{e}-2$ & $0.698 \mathrm{e}-3$ & $-1.24 \mathrm{e}-5$ \\
$3 / 2$ & $0.649 \mathrm{e}-2$ & $0.600 \mathrm{e}-3$ & $-0.578 \mathrm{e}-5$ \\
2 & $0.605 \mathrm{e}-2$ & $0.521 \mathrm{e}-3$ & $-2.12 \mathrm{e}-6$ \\
\hline \hline
\end{tabular}

TABLE VIII. Values of the coefficients $\tilde{d}_{j}, j=2,3,4$, for the scheme-independent expansion of $\beta_{\mathrm{IR}}^{\prime}$, Eq. (6.2), in an $\mathrm{SU}(3)$ gauge theory with fermions in the fundamental and adjoint representations, for illustrative values of $N_{F}$.

\begin{tabular}{llcc}
\hline \hline$N_{F}$ & $\tilde{d}_{2}$ & $\tilde{d}_{3}$ & $\tilde{d}_{4}$ \\
\hline 0 & 0.1975 & 0.117 & 0.0265 \\
4 & 0.215 & 0.139 & 0.0313 \\
8 & 0.236 & 0.168 & 0.0358 \\
10 & 0.249 & 0.186 & 0.0374 \\
12 & 0.262 & 0.206 & 0.0379 \\
\hline \hline
\end{tabular}

$$
\begin{gathered}
\tilde{d}_{2}=\frac{2^{5} T_{f^{\prime}}^{2}}{3^{2} \mathcal{D}_{f^{\prime}}} \\
\tilde{d}_{3}=\frac{2^{7} T_{f^{\prime}}^{3}\left(5 C_{A}+3 C_{f^{\prime}}\right)}{3^{3} \mathcal{D}_{f^{\prime}}^{2}},
\end{gathered}
$$

and similarly for $\tilde{d}_{4}$.

\section{RESULTS FOR $\beta_{\text {IR }}^{\prime}$ IN A THEORY WITH FERMIONS IN THE FUNDAMENTAL AND ADJOINT REPRESENTATIONS OF SU $\left(N_{c}\right)$}

In this section we discuss the special case of our general calculation of $\beta_{\mathrm{IR}}^{\prime}$ for an $\mathrm{SU}\left(N_{c}\right)$ theory with $N_{f}$ fermions in the fundamental representation and $N_{\text {Adj }}$ fermions in the adjoint representation (i.e., the FA theory). We write Eqs. (1.8) and (1.9) as

$$
\beta_{\mathrm{IR}}^{\prime}=\sum_{j=2}^{\infty} d_{j} \Delta_{F}^{j}
$$

and

$$
\beta_{\mathrm{IR}}^{\prime}=\sum_{j=2}^{\infty} \tilde{d}_{j} \Delta_{A d j}^{j}
$$

where $\Delta_{F}$ and $\Delta_{A d j}$ were given explicitly in Eqs. (4.21)(4.24). 
We calculate

$$
\begin{gathered}
d_{2}=\frac{2^{4}}{3^{2}\left[25 N_{c}^{2}-11+4 N_{A d j}\left(N_{c}^{2}+1\right)\right]} \\
\tilde{d}_{2}=\frac{2^{5} N_{c}^{3}}{3^{2}\left[18 N_{c}^{3}-N_{F}\left(N_{c}^{2}+1\right)\right]} \\
d_{3}=\frac{2^{5}\left(13 N_{c}^{2}-3\right)}{3^{3} N_{c}\left[25 N_{c}^{2}-11+4 N_{A d j}\left(N_{c}^{2}+1\right)\right]^{2}}
\end{gathered}
$$

$$
\tilde{d}_{3}=\frac{2^{10} N_{c}^{6}}{3^{3}\left[18 N_{c}^{3}-N_{F}\left(N_{c}^{2}+1\right)\right]^{2}}
$$$$
d_{4}=\frac{2^{4}\left[d_{4,0}+d_{4,1} N_{A d j}+d_{4,2} N_{A d j}^{2}+d_{4,3} N_{A d j}^{3}\right]}{3^{5} N_{c}^{2}\left[25 N_{c}^{2}-11+4 N_{A d j}\left(N_{c}^{2}+1\right)\right]^{5}} .
$$

$$
\begin{aligned}
d_{4,0}= & 366782 N_{c}^{8}-865400 N_{c}^{6}+1599316 N_{c}^{4}-571516 N_{c}^{2}-3993 \\
+ & \zeta_{3} N_{c}^{2}\left[-660000 N_{c}^{6}+765600 N_{c}^{4}-2241888 N_{c}^{2}+894432\right] \\
d_{4,1}= & 18416 N_{c}^{8}+346944 N_{c}^{6}-756920 N_{c}^{4}+530256 N_{c}^{2}+2904 \\
+ & \zeta_{3} N_{c}^{2}\left[28800 N_{c}^{6}+372096 N_{c}^{4}+1026432 N_{c}^{2}-975744\right] \\
d_{4,2}= & 2^{4}\left(N_{c}^{2}+1\right)\left[-3161 N_{c}^{6}+10589 N_{c}^{4}-10155 N_{c}^{2}-33\right. \\
& \left.+\zeta_{3} N_{c}^{2}\left(3744 N_{c}^{4}-13248 N_{c}^{2}+22176\right)\right] \\
d_{4,3}= & 2^{8} N_{c}^{2}\left(N_{c}^{2}+1\right)^{2}\left[-23 N_{c}^{2}+65+\zeta_{3}\left(24 N_{c}^{2}-168\right)\right]
\end{aligned}
$$

and

$$
\tilde{d}_{4}=\frac{2^{3} N_{c}^{5}\left[\hat{d}_{4,0}+\hat{d}_{4,1} N_{F}+\hat{d}_{4,2} N_{F}^{2}+\hat{d}_{4,3} N_{F}^{3}\right]}{3^{5}\left[18 N_{c}^{3}-N_{F}\left(N_{c}^{2}+1\right)\right]^{5}},
$$

where

$$
\begin{gathered}
\hat{d}_{4,0}=3^{3} N_{c}^{8}\left(46871 N_{c}^{2}+85248\right) \\
\hat{d}_{4,1}=36 N_{c}^{3}\left[1287 N_{c}^{6}-23350 N_{c}^{4}-1961 N_{c}^{2}+276+\zeta_{3} N_{c}^{4}\left(-6912 N_{c}^{2}+16128\right)\right] \\
\hat{d}_{4,2}=4\left(N_{c}^{2}+1\right)\left[-5153 N_{c}^{6}+18113 N_{c}^{4}-747 N_{c}^{2}-141+\zeta_{3} N_{c}^{4}\left(6912 N_{c}^{2}-32256\right)\right] \\
\hat{d}_{4,3}=2^{5} N_{c}\left(N_{c}^{2}+1\right)^{2}\left[23 N_{c}^{2}-65+\zeta_{3}\left(-24 N_{c}^{2}+168\right)\right] .
\end{gathered}
$$

If $N_{A d j}=0$, then $d_{2}, d_{3}$, and $d_{4}$ reduce to our previous results in, respectively, Eqs. (5.14), (5.15), and (5.16) of [13]. Similarly, if $N_{F}=0$, then $\tilde{d}_{2}, \tilde{d}_{3}$, and $\tilde{d}_{4}$ reduce to our previous results in, respectively, Eqs. (5.59), (5.60), and (5.61) of [13]. The agreement of these reductions of $d_{j}$ for $N_{\text {Adj }}=0$ and of $\tilde{d}_{j}$ for $N_{F}=0$ with our results in [13] for $j=1,2,3$ serves as a check on our present calculations. As was discussed in $[13,15]$, these coefficients have the leading large- $N_{c}$ dependence

$$
d_{j} \sim N_{c}^{-j} \quad \text { as } N_{c} \rightarrow \infty
$$

and

$$
\tilde{d}_{j} \sim N_{c}^{0} \quad \text { as } N_{c} \rightarrow \infty
$$

In Tables VII and VIII we list the values of $d_{j}$ and $\tilde{d}_{j}$ with $j=1,2,3$ for illustrative SU(3) FA theories. In Table IX we present our scheme-independent calculations of $\beta_{\mathrm{IR}}^{\prime}$ to order $O\left(\Delta_{F}^{p}\right)$ via the expansion (6.1) and to $O\left(\Delta_{A d j}^{p}\right)$ via the expansion (6.2), with $p=1,2,3$, where $\Delta_{F}$ and $\Delta_{A d j}$ were defined in Eqs. (4.21)-(4.24). These are denoted $\beta_{\mathrm{IR}, \Delta_{F}^{p}}^{\prime}$ and $\beta_{\mathrm{IR}, \Delta_{A d j}^{p}}^{\prime}$, respectively. Graphically, in the first quadrant of $\mathbb{R}^{2}$ defined by $\left(N_{F}, N_{a d j}\right)$ (formally generalized to nonnegative real numbers), the series (6.1) is an expansion in a leftward horizontal direction from the $b_{1}=0$ line toward a 
TABLE IX. Values of $\beta_{\mathrm{IR}}^{\prime}$ as calculated to order $O\left(\Delta_{f}^{p}\right)$ via Eq. (6.1), denoted $\beta_{\mathrm{IR}, \Delta_{F}^{p}}^{\prime}$ and to order $O\left(\Delta_{A d j}^{p}\right)$ via Eq. (6.2), denoted $\beta_{\mathrm{IR}, \Delta_{A d j}^{p}}^{\prime}$, with $p=2,3,4$, in an $\mathrm{SU}(3)$ gauge theory with $N_{F}$ fermions in the fundamental $(F)$ representation and $N_{A d j}$ fermions in the adjoint $(A d j)$ representation. Here, half-integral values of $N_{A d j}$ refer to theories with $2 N_{A d j}$ copies of Majorana fermions in the adjoint representation. The notation ae-n means $a \times 10^{-n}$.

\begin{tabular}{lllllll}
\hline \hline$\left(N_{F}, N_{A d j}\right)$ & $\beta_{\mathrm{IR}, \Delta_{F}^{2}}^{\prime}$ & $\beta_{\mathrm{IR}, \Delta_{A d j}^{2}}^{\prime}$ & \multicolumn{1}{c}{$\beta_{\mathrm{IR}, \Delta_{F}^{3}}^{\prime}$} & $\beta_{\mathrm{IR}, \Delta_{d d j}^{3}}^{\prime}$ & $\beta_{\mathrm{IR}, \Delta_{F}^{4}}^{\prime}$ & $\beta_{\mathrm{IR}, \Delta_{A d j}^{4}}^{\prime}$ \\
\hline$(8,1 / 2)$ & 0.230 & 0.199 & 0.367 & 0.328 & 0.344 & 0.353 \\
$(8,1)$ & $4.374 \mathrm{e}-2$ & $4.105 \mathrm{e}-2$ & $5.465 \mathrm{e}-2$ & $5.32 \mathrm{e}-2$ & $5.42 \mathrm{e}-2$ & $5.43 \mathrm{e}-2$ \\
$(10,0)$ & 0.351 & 0.292 & 0.621 & 0.528 & 0.538 & 0.579 \\
$(10,1 / 2)$ & 0.0931 & 0.0846 & 0.128 & 0.1215 & 0.125 & 0.126 \\
$(10,1)$ & $1.75 \mathrm{e}-3$ & $1.73 \mathrm{e}-3$ & $1.837 \mathrm{e}-3$ & $1.8345 \mathrm{e}-3$ & $1.8363 \mathrm{e}-3$ & $1.8361 \mathrm{e}-3$ \\
$(12,0)$ & 0.168 & 0.1475 & 0.258 & 0.235 & 0.239 & 0.247 \\
$(12,1 / 2)$ & $1.71 \mathrm{e}-2$ & $1.64 \mathrm{e}-2$ & $1.987 \mathrm{e}-2$ & $1.962 \mathrm{e}-2$ & $1.975 \mathrm{e}-2$ & $1.977 \mathrm{e}-2$ \\
\hline \hline
\end{tabular}

given point $\left(N_{F}, N_{A d j}\right)$ in the NACP, while the series (6.1) is an expansion inward in a downward vertical direction from the $b_{1}=0$ line toward this point $\left(N_{F}, N_{A d j}\right)$. Since these are two alternate expansions for the same quantity, one expects that as the maximal power $p$ in the series increases, they should yield similar values, and we see that this expectation is satisfied by our results at the highest order, $p=3$, as listed in Table IX. The agreement between the two series is best when the $\left(N_{F}, N_{A d j}\right)$ theory is near to the upper end of the non-Abelian Coulomb phase, since in this case the expansion parameters $\Delta_{F}$ and $\Delta_{A d j}$ are the smallest. Some explicit examples that demonstrate this accuracy are provided by the following fractional differences:

$$
\begin{aligned}
& \mathrm{SU}(3),\left(N_{F}, N_{A d j}\right)=(10,1) \\
\Rightarrow & \frac{\left|\beta_{\mathrm{IR}, \Delta_{F}^{4}}^{\prime}-\beta_{\mathrm{IR}, \Delta_{A d j}^{4}}^{\prime}\right|}{\beta_{\mathrm{IR}, \Delta_{F}^{4}}^{\prime}}=2.2 \times 10^{-5}
\end{aligned}
$$

and

$$
\begin{aligned}
\operatorname{SU}(3),\left(N_{F}, N_{A d j}\right) & =\left(12, \frac{1}{2}\right) \\
\Rightarrow & \frac{\left|\beta_{\mathrm{IR}, \Delta_{F}^{4}}^{\prime}-\beta_{\mathrm{IR}, \Delta_{A d j}^{4}}^{\prime}\right|}{\beta_{\mathrm{IR}, \Delta_{F}^{4}}^{\prime}}=1.0 \times 10^{-3} .
\end{aligned}
$$

\section{CONCLUSIONS}

In this paper, generalizing our previous work, we have considered an asymptotically free gauge theory with gauge group $G$ and two different fermion representations, with the property that it exhibits an infrared fixed point such that the infrared theory is in a non-Abelian Coulomb phase. Specifically, we have considered a theory with $N_{f}$ fermions transforming according to a representation $R$ of $G$ and $N_{f^{\prime}}$ fermions transforming according to a different representation, $R^{\prime}$. We have calculated scheme-independent series expansions of the anomalous dimensions of gauge invariant fermion bilinears and the derivative $\beta_{\mathrm{IR}}^{\prime}$ evaluated at the IR fixed point in the respective expansion parameters $\Delta_{f}$ and $\Delta_{f^{\prime}}$. As an explicit application, we have presented calculations for an $\mathrm{SU}\left(N_{c}\right)$ theory with $N_{F}$ fermions in the fundamental representation and $N_{A d j}$ fermions in the adjoint representation. Our results for scheme-independent expansions of gauge-invariant fermion bilinears extend up to $O\left(\Delta_{F}^{3}\right)$ and $O\left(\Delta_{\text {Adj }}^{3}\right)$, while our results for $\beta_{\mathrm{IR}}^{\prime}$ extend up to $O\left(\Delta_{F}^{4}\right)$ and $O\left(\Delta_{A d j}^{4}\right)$. These results provide further information about the properties of these conformal field theories. To the extent that the transition from the lower part of the non-Abelian Coulomb phase to the quasi-conformal regime in the variables $\left(N_{f}, N_{f^{\prime}}\right)$ is continuous and our finite-order perturbative calculations in the lower part of the non-Abelian Coulomb phase are sufficiently accurate, our present results can also be useful for the investigation of quasi-conformal theories with possible relevance to ultraviolet completions of the Standard Model that address the Higgs mass naturalness problem and the generational hierarchy of SM fermion masses.

\section{ACKNOWLEDGMENTS}

This research was supported in part by the Danish National Research Foundation Grant No. DNRF90 to $\mathrm{CP}^{3}$-Origins at SDU (T. A. R.) and by the U.S. NSF Grant No. NSF-PHY-16-1620628 (R. S.)

\section{APPENDIX: GROUP INVARIANTS}

In this appendix we discuss some relevant grouptheoretic quantities. Let us denote the generators of the Lie algebra of the gauge group $G$, in the representation $R$, as $T_{R}^{a}$, with $1 \leq a \leq d_{A}$, where $d_{A}$ is the order of the group. These generators satisfy the commutation relations

$$
\left[T_{R}^{a}, T_{R}^{b}\right]=i f^{a b c} T_{R}^{c},
$$


where the $f^{a b c}$ are the associated structure constants of this Lie algebra. Here and elsewhere, a sum over repeated indices is understood. We denote the dimension of a given representation $R$ as $d_{R}=\operatorname{dim}(R)$. In particular, we denote the adjoint representation by $A$, with the dimension $d_{A}$ equal to the number of generators of the group, i.e., the order of the group. The trace invariant is given by

$$
\operatorname{Tr}_{R}\left(T_{R}^{a} T_{R}^{b}\right)=T(R) \delta_{a b} .
$$

The quadratic Casimir invariant $C_{2}(R)$ is defined by

$$
T_{R}^{a} T_{R}^{a}=C_{2}(R) I
$$

where $I$ is the $d_{R} \times d_{R}$ identity matrix. For a fermion $f$ transforming according to a representation $R$, we often use the equivalent compact notation $T_{f} \equiv T(R)$ and $C_{f} \equiv C_{2}(R)$. We also use the notation $C_{A} \equiv C_{2}(A) \equiv C_{2}(G)$. The invariants $T(R)$ and $C_{2}(R)$ satisfy the relation $C_{2}(R) d_{R}=$ $T(R) d_{A}$. For $G=\mathrm{SU}\left(N_{c}\right), C_{A}=N_{c}$ and for $R$ equal to the fundamental representation, $T(R)=1 / 2$ and $C_{2}(R)=$ $\left(N_{c}^{2}-1\right) /\left(2 N_{c}\right)$.

At the four-loop and five-loop level, one encounters traces of quartic products of the Lie algebra generators. For a given representation $R$ of $G$,

$$
\begin{aligned}
d_{R}^{a b c d}= & \frac{1}{3 !} \operatorname{Tr}_{R}\left[T _ { a } \left(T_{b} T_{c} T_{d}+T_{b} T_{d} T_{c}+T_{c} T_{b} T_{d}\right.\right. \\
& \left.\left.+T_{c} T_{d} T_{b}+T_{d} T_{b} T_{c}+T_{d} T_{c} T_{b}\right)\right] .
\end{aligned}
$$

As with the quadratic invariants, for a fermion $f$ in the representation $R$ of $G$, we often use the notation $d_{R}^{a b c d} \equiv d_{f}^{a b c d}$. In this context, for $R=A d j$, we use $d_{R}^{a b c d}=d_{A}^{a b c d}$. The quantities that appear in the anomalous dimensions and derivative of the beta function $\beta_{\mathrm{IR}}^{\prime}$ that we calculate are products of these $d_{R}^{a b c d}$ of the form $d_{R}^{a b c d} d_{R^{\prime}}^{a b c d} \equiv d_{f}^{a b c d} d_{f^{\prime}}^{a b c d}$, summed over the group indices $a, b, c, d$. For further discussion of these, with references to the literature, see $[16,44]$ and references therein.
[1] Taking the fermions to be massless does not incur any loss of generality, because a fermion with a nonzero mass $m_{0}$ would be integrated out of the low-energy effective field theory at Euclidean momentum scales $\mu<m_{0}$, and hence would be irrelevant to the properties of the theory at the IRFP of interest here. We note also that our theories do not contain any scalar fields.

[2] T. A. Ryttov and F. Sannino, Int. J. Mod. Phys. A 25, 4603 (2010).

[3] T. A. Ryttov and R. Shrock, Phys. Rev. D 81, 116003 (2010).

[4] J. Polchinski, Nucl. Phys. B303, 226 (1988); J.-F. Fortin, B. Grinstein, and A. Stergiou, J. High Energy Phys. 01 (2013) 184; A. Dymarsky, Z. Komargodski, A. Schwimmer, and S. Thiessen, J. High Energy Phys. 10 (2015) 171, and references therein.

[5] T. A. Ryttov and R. Shrock, Phys. Rev. D 83, 056011 (2011).

[6] C. Pica and F. Sannino, Phys. Rev. D 83, 035013 (2011).

[7] R. Shrock, Phys. Rev. D 87, 105005 (2013); 87, 116007 (2013).

[8] T. A. Ryttov and R. Shrock, Phys. Rev. D 94, 105015 (2016).

[9] T. Banks and A. Zaks, Nucl. Phys. B196, 189 (1982).

[10] G. Grunberg, Phys. Rev. D 46, 2228 (1992); E. Gardi and M. Karliner, Nucl. Phys. B529, 383 (1998); E. Gardi and G. Grunberg, J. High Energy Phys. 03 (1999) 024.

[11] T. A. Ryttov, Phys. Rev. Lett. 117, 071601 (2016).

[12] T. A. Ryttov and R. Shrock, Phys. Rev. D 94, 105014 (2016).

[13] T. A. Ryttov and R. Shrock, Phys. Rev. D 94, 125005 (2016).

[14] T. A. Ryttov and R. Shrock, Phys. Rev. D 95, 085012 (2017).

[15] T. A. Ryttov and R. Shrock, Phys. Rev. D 95, 105004 (2017).

[16] T. A. Ryttov and R. Shrock, Phys. Rev. D 96, 105015 (2017).

[17] T. A. Ryttov and R. Shrock, Phys. Rev. D 97, 025004 (2018).
[18] T. A. Ryttov and R. Shrock, Phys. Rev. D 96, 105018 (2017); 97, 065020 (2018).

[19] T. A. Ryttov and R. Shrock, Phys. Rev. D 97, 016020 (2018).

[20] For recent reviews of these lattice simulations, see, e.g., talks in the Lattice for BSM 2017 Workshop at http://wwwhep.colorado.edu/(tilde)eneil/lbsm17; Lattice-2017 at http:// wpd.ugr.es/(tilde)lattice2017; [21]; and Lattice-2018 at https://web.pa.msu.edu/conf/Lattice2018. At present, there is not a complete consensus among lattice groups about the respective values of $N_{f, c r}$ for various theories.

[21] Simons Workshop on Continuum and Lattice Approaches to the Infrared Behavior of Conformal and Quasiconformal Gauge Theories, 2018, T. A. Ryttov and R. Shrock, organizers, http://scgp.stonybrook.edu/archives/21358.

[22] Furthermore, in the case where $G=\mathrm{SU}\left(N_{c}\right)$ and the fermions are in the fundamental representation, one can take the limits $N_{c} \rightarrow \infty$ and $N_{f} \rightarrow \infty$ with $r=N_{f} / N_{c}$ fixed and finite. In this case, $\alpha_{\mathrm{IR}}$ can be made arbitrarily small.

[23] Some authors use the opposite sign convention for the anomalous dimension, writing $D_{\mathcal{O}}=D_{\mathcal{O} \text {,free }}+\gamma_{\mathcal{O}}$. Our sign convention is the same as the convention used in lattice gauge theory literature.

[24] J. A. Gracey, Phys. Lett. B 488, 175 (2000).

[25] G. 't Hooft, Nucl. Phys. B61, 455 (1973).

[26] W. A. Bardeen, A. J. Buras, D. W. Duke, and T. Muta, Phys. Rev. D 18, 3998 (1978).

[27] D. J. Gross, in Methods in Field Theory, Les Houches 1975, edited by R. Balian and J. Zinn-Justin (North Holland, Amsterdam, 1976), p. 141.

[28] D. J. Gross and F. Wilczek, Phys. Rev. Lett. 30, 1343 (1973); H. D. Politzer, Phys. Rev. Lett. 30, 1346 (1973). 
[29] W. E. Caswell, Phys. Rev. Lett. 33, 244 (1974); D. R. T. Jones, Nucl. Phys. B75, 531 (1974).

[30] O. V. Tarasov, A. A. Vladimirov, and A. Yu. Zharkov, Phys. Lett. 93B, 429 (1980); S. A. Larin and J. A. M. Vermaseren, Phys. Lett. B 303, 334 (1993).

[31] T. van Ritbergen, J. A. M. Vermaseren, and S. A. Larin, Phys. Lett. B 400, 379 (1997).

[32] F. Herzog, B. Ruijl, T. Ueda, J. A. M. Vermaseren, and A. Vogt, J. High Energy Phys. 02 (2017) 090.

[33] P. A. Baikov, K. G. Chetyrkin, and J. H. Kühn, Phys. Rev. Lett. 118, 082002 (2017).

[34] M. F. Zoller, J. High Energy Phys. 10 (2016) 118.

[35] K. G. Chetyrkin, Phys. Lett. B 404, 161 (1997); J. A. M. Vermaseren, S. A. Larin, and T. van Ritbergen, Phys. Lett. B 405, 327 (1997).

[36] P. A. Baikov, K. G. Chetyrkin, and J.H. Kühn, J. High Energy Phys. 10 (2014) 076; 04 (2017) 119.

[37] K. G. Chetyrkin and M. F. Zoller, J. High Energy Phys. 06 (2017) 074.

[38] J. A. Gracey, T. A. Ryttov, and R. Shrock, Phys. Rev. D 97, 116018 (2018).

[39] In principle, for certain $G, R$, and $R^{\prime}$, it might be possible for there to be an intermediate phase between the (deconfined) non-Abelian Coulomb phase and the QCD-like phase with confinement and $\mathrm{S} \chi \mathrm{SB}$ with the property that in this intermediate phase there is confinement but no $\mathrm{S} \chi \mathrm{SB}$. A necessary but not sufficient condition for this would be that the 't Hooft anomalymatching conditions are satisfied. This possibility will not be directly relevant for our calculations in the NACP and so we will not pursue it here.

[40] V. Ayyar, T. DeGrand, M. Golterman, D. Hackett, W. I. Jay, E. T. Neil, Y. Shamir, and B. Svetitsky, Phys. Rev. D 97, 074505 (2018).

[41] V. Ayyar, T. DeGrand, D. Hackett, W. I. Jay, E. T. Neil, Y. Shamir, and B. Svetitsky, Phys. Rev. D 97, 114505 (2018); 97, 114502 (2018).

[42] S. L. Adler, J. C. Collins, and A. Duncan, Phys. Rev. D 15, 1712 (1977); J. C. Collins, A. Duncan, and S. Joglekar, Phys. Rev. D 16, 438 (1977); N. K. Nielsen, Nucl. Phys. B120, 212 (1977).See also H. Kluberg-Stern and J.-B. Zuber, Phys. Rev. D 12, 467 (1975).

[43] See, e.g., S. S. Gubser, A. Nellore, S. S. Pufu, and E. D. Rocha, Phys. Rev. Lett. 101, 131601 (2008); see also M. Kurachi, S. Matsuzaki, and K. Yamawaki, Phys. Rev. D 90, 055028 (2014); R. J. Crewther and L. C. Tunstall, Phys. Rev. D 91, 034016 (2015).

[44] T. van Ritbergen, A. N. Schellekens, and J. A. M. Vermaseren, Int. J. Mod. Phys. A 14, 41 (1999). 